\title{
Deconstructing the molecular genetics behind the PINK1/Parkin axis in Parkinson's disease using Drosophila melanogaster as a model organism
}

\author{
Suchita Ganesan and Venkatachalam Deepa Parvathi ${ }^{*}$ (1)
}

\begin{abstract}
Background: Parkinson's disease (PD) is a multifactorial neurodegenerative disorder marked by the death of nigrostriatal dopaminergic neurons in response to the compounding effects of oxidative stress, mitochondrial dysfunction and protein aggregation. Transgenic Drosophila models have been used extensively to decipher the underlying genetic interactions that exacerbate neural health in PD. Autosomal recessive forms of the disease have been linked to mutations in the serine/threonine kinase PINK1(PTEN-Induced Putative Kinase 1) and E3 ligase Parkin, which function in an axis that is conserved in flies. This review aims to probe the current understanding of PD pathogenesis via the PINK1/Parkin axis while underscoring the importance of several molecular and pharmacologic rescues brought to light through studies in Drosophila.

Main body: Mutations in PINK1 and Parkin have been shown to affect the axonal transport of mitochondria within dopaminergic neurons and perturb the balance between mitochondrial fusion/fission resulting in abnormal mitochondrial morphology. As per studies in flies, ectopic expression of Fwd kinase and Atg-1 to promote fission and mitophagy while suppressing fusion via MUL1 E3 ligase may aid to halt mitochondrial aggregation and prolong the survival of dopaminergic neurons. Furthermore, upregulation of Hsp70/Hsp90 chaperone systems (Trap1, CHIP) to target misfolded mitochondrial respiratory complexes may help to preserve their bioenergetic capacity. Accumulation of reactive oxygen species as a consequence of respiratory complex dysfunction or antioxidant enzyme deficiency further escalates neural death by inducing apoptosis, lipid peroxidation and DNA damage. Fly studies have reported the induction of canonical Wnt signalling to enhance the activity of transcriptional co-activators (PGC1a, FOXO) which induce the expression of antioxidant enzymes. Enhancing the clearance of free radicals via uncoupling proteins (UCP4) has also been reported to ameliorate oxidative stress-induced cell death in PINK1/Parkin mutants.

Conclusion: While these novel mechanisms require validation through mammalian studies, they offer several explanations for the factors propagating dopaminergic death as well as promising insights into the therapeutic importance of transgenic fly models in PD.
\end{abstract}

Keywords: Parkinson's disease, PINK1, Parkin, Drosophila melanogaster, Mitochondrial dynamics, Apoptosis, Oxidative stress, Protein misfolding

*Correspondence: deepaparvathi@sriramachandra.edu.in; deepakoushik305@gmail.com

Department of Biomedical Sciences, Faculty of Biomedical Sciences and Technology, Sri Ramachandra Institute of Higher Education and Research, Chennai, Tamil Nadu 600116, India

\section{Background}

Parkinson's disease (PD) is a progressive neurodegenerative disorder that occurs due to the depletion of dopaminergic neurons within the substantia nigra pars compacta 
(SNpc) of the basal ganglia, a complex network of nuclei that regulate movement [1]. The disease clinically manifests through motor dysfunctions such as bradykinesia, postural instability, tremor and rigidity with the onset of motor symptoms following an asymptomatic period marked by hyposmia, constipation or disturbances in sleep patterns [2]. There still exists a great variation in the pattern of progression with increasing reports of overlapping pathophysiology, symptoms presented, and interplay between environmental and genetic factors thus classifying PD as a heterogenous movement disorder [2, 3]. In developed countries, the prevalence of PD has increased over the past two decades, with prospective populationbased studies reporting standardized incidence rates of 8-18 per 100,000 person-years [4]. The tendency for higher prevalence in developed countries has been attributed to the increased exposure towards environmental toxins as a consequence of extensive industrialization [5]. Although the vast majority of cases are considered to be sporadic, over 20 gene loci (termed PARK loci) have been implicated in heritable forms of the disease. Specifically, mutations in $\alpha$-synuclein (SNCA/PARK1), leucine-rich repeat kinase 2 (LRRK2/PARK8) and vacuolar protein sorting ortholog 35 (VPS35/PARK17) have been associated with autosomal dominant PD, whereas Parkin (PARK2), PTEN-induced kinase 1 (PINK1/PARK6) and protein deglycase (DJ-1/PARK7) mutations have been linked to autosomal recessive forms [6].

While the genetic landscape of PD has been rigorously explored in western populations, there have been significantly fewer large-scale studies emerging from Asian countries $[5,7,8]$. Lower percentages of aged populations, lack of awareness of symptoms amongst affected individuals and mis-diagnosis are the key factors behind low incidence rates throughout Asia. Smoking, pesticide exposure and caffeine intake are a few risk factors well established across all populations [5]. Nonetheless, with PD being a disorder that predominantly targets individuals of a higher age bracket (70-80), the disease burden of PD and similar age-dependent neurological disorders is comparatively higher in western countries [9].

Autosomal recessive forms of PD manifest relatively early on (under 50 years of age or juvenile onset if under 30) with patients presenting classic motor symptoms such as dyskinesias and dystonia. In such patients, the response to levodopa therapy is often conflated with the increased occurrence of dyskinesias, therefore escalating motor damage [10]. Taking these features into account, there is an exigent need to deconstruct the factors precipitating neural death. Concerning the pathogenic basis behind PD, the deterioration of neural health has been attributed to the compounding effects of protein aggregation, mitochondrial dysfunction, oxidative assault and cascading neuroinflammatory reactions [1]. Moreover, a notable amount of research has sought to uncover the roles of the aforementioned genes in the abrogation of these mechanisms [11]. PINK1 and Parkin have shown to be highly interactive functioning as an axis that regulates mitochondrial biogenesis, apoptosis, oxidative stress and protein misfolding [12-14]. Multiple organisms have been utilised to decipher the nature of this axis. The fruit fly Drosophila melanogaster, in particular, has been considered as a robust model universally employed to investigate neurodegenerative diseases such as PD due to its short lifespan, ability to exhibit locomotor defects and replicate Lewy body pathology [15]. The latter stands as a key feature that distinguishes flies from mammalian models which often fail to replicate pathological hallmarks of PD, thus making them less suitable for studying pathogenic mechanisms [16]. Drosophila maintains homologues for almost $77 \%$ of mammalian disease causing genes and possesses a highly organized dopaminergic neuron system allowing for an efficient understanding of the molecular events foregoing neural degeneration. Some of the techniques used to generate models of mutant flies have involved the application of GAL4/UAS drivers, RNA interference (RNAi) and more recently, CRISPR/Cas9 genome editing technology. GAL4/UAS drivers have been designed to study the mis-expression of transgenes in a tissue specific manner without compromising fly survival while RNA interference has been used to study the nature of recessive genes by inducing gene knockdown or loss of function [15, 17, 18]. So far, Drosophila PINK1/Parkin models have been extensively studied to provide empirical evidence supporting the link between mitochondrial dysfunction, oxidative stress and neural death [15]. This review aims to provide a comprehensive analysis of the behaviour of the PINK1/Parkin axis while highlighting how studies in flies have yielded important insights into possible molecular and pharmacologic rescues suitable for therapeutic exploitation in patients with autosomal recessive PD.

\section{Drosophila melanogaster: a robust model for Parkinson's disease}

Multiple mammalian (rodents, non-human primates) and non-mammalian (Drosophila melanogaster (fruit fly), Danio rerio (zebrafish), Caenorhabditis (C.) elegans, Oryzias latipes (medaka fish)) models have been utilized to study familial forms of PD. Non-mammalian models have been viciously employed due to their low cost, short lifespan, simple cultivation and ease of genetic manipulation owed to their small genome size [19]. By implementing the use of GAL4/UAS drivers and RNAi, studying the effects of tissue specific gene overexpression/silencing on disease phenotypes is relatively simple [15, 17-19]. 
Drosophila models are specifically capable of producing a wide range of distinct motor phenotypes (walking/ flying/climbing deficits) consistent with patterns of agedependent dopaminergic cell loss $[15,20]$. Unlike other non-mammalian models, the pathways of dopamine metabolism are also highly conserved between flies and humans [21]. Homologues for several PD causing genes such as PINK1, Parkin, DJ-1, VPS35 and LRRK2 are encoded by the fly genome. Although flies lack a homologue for $S N C A$, overexpression of human wild type SNCA and disease-causing mutant forms in Drosophila induces dopaminergic death, motor and non-motor PD phenotypes [22].

While mammalian models bear greater physiological and genetic similarities to humans, they hold certain limitations [16]. Firstly, the generation of transgenic rodents requires additional effort to counteract issues of transgene silencing, leaky expression and unexpected mutations due to random integration [23]. Transgenic rodents have also presented several inconsistencies in key pathological hallmarks of PD. These include mild defects in mitochondrial morphology, faint or complete absence of dopaminergic cell loss in the SNpc as well as a lack of Lewy body inclusions [19]. The reasons for these inconsistencies have been attributed to the possibility of alternative mechanisms of genetic compensation and the fact that pathology may be additionally dependent on the exposure to environmental toxins [16, 24]. To counteract this issue, some have proposed that inducing genetic defects alongside exposure to environmental toxins might help to produce more pronounced pathology. However, few studies have confirmed the validity of this method [25].

While there is still no genetic model that strongly displays all pathological hallmarks of PD, such glaring issues in rodent models are hard to overlook. For these reasons, Drosophila serves as comparatively ideal model to study the genetic and molecular underpinnings of PD pathogenesis [19].

\section{Parkin- E3 ubiquitin ligase}

Mutations in the PARK2 locus account for the majority of autosomal recessive juvenile cases of PD [26, 27]. The PARK2 locus located on the long arm of chromosome 6 encodes the protein Parkin which belongs to the family of E3 ubiquitin (Ub) ligases [28]. Parkin possesses a $\mathrm{N}$-terminal ubiquitin like domain (UBL), three RING (really interesting new gene) domains (RING0, RING1, RING2) separated by an in-between RING (IBR) domain and an autoinhibitory REP (repressor element of parkin) region. RING1, IBR and RING2 fold to form a RING-inbetween-RING (RBR) segment. Following the activation of E2 (ubiquitin conjugating enzyme) by E1 (ubiquitin activating enzyme), Parkin (a RBR ligase) catalyses the transfer of ubiquitin from the cysteine residue of E2 to its own cysteine residue on its RING2 domain. Ub-bound Parkin ubiquitinates specific target proteins which may be degraded by a proteasome (ubiquitin proteasome system/UPS) or used to alter cellular functions [29, 30]. Parkin substrates take on different downstream processes depending on the type of ubiquitin linkage they possess [31]. In case of PD, mutations in Parkin have been shown to abolish its ability to target proteins for ubiquitylation and proteasomal degradation thus precipitating dopaminergic cell loss [26, 27].

\section{PINK1 serine/threonine kinase}

Mutations in the PARK6 locus are also significantly responsible for triggering autosomal recessive juvenile PD [32]. Parkin interacts with the mitochondrial serine/threonine kinase PTEN-induced putative kinase 1 (PINK1), encoded by the PARK6 locus located on the short arm of chromosome 1 [28]. PINK1 possesses a $\mathrm{N}$-terminal mitochondrial targeting sequence, an $\alpha$-helix transmembrane domain, a series of insertion-linked-beta strands that make up the main kinase domain followed by a non-catalytic C-terminal domain [33]. Following stressinduced depolarization of the outer mitochondrial membrane, PINK1 ceases to undergo $\mathrm{N}$-terminal cleavage via the inner membrane protease PARL (presenilin-associated rhomboid-like protein) for import to the inner mitochondrial membrane and instead remains embedded in the outer membrane [34]. Here, PINK1 stabilizes through autophosphorylation of its kinase domains and proceeds to phosphorylate cytosolic Parkin on serine (Ser 65) or threonine (Thr 175) residues to induce its translocation to the mitochondria [35-37]. While PINK1 is primarily localized to the mitochondria, $\mathrm{N}$-terminal cleaved fragments are also localized within the cytosol upon Cdc37/ Hsp90 chaperone mediated processing [38].

Mutations in PINK1 have been found to result in loss of kinase activity due to conformational changes in its catalytic domains, and reduced translocation to the mitochondrial outer membrane due to poor interaction with import-export machinery [39]. Consequently, loss of PINK1 mediated phosphorylation impedes the translocation of Parkin for mitochondrial quality control.

The cellular consequences of PINK1/Parkin dysfunction with regard to PD pathology are complex and will be discussed in the subsequent sections.

\section{The PINK1/Parkin axis in Drosophila}

Both Parkin and PINK1 are functionally conserved in Drosophila melanogaster. Overexpression of Drosophila parkin can rescue pink1 null phenotypes but not vice versa confirming that pink1 functions upstream of 
parkin, as seen in humans $[28,40]$. The serine/threonine kinase domain of Drosophila pink1 shares 43\% amino acid sequence homology with human PINK1 [40]. RNAi knockdown of fly pink1 has been observed to induce PD-like phenotypes such as abnormal wing posture, indirect flight muscle degeneration, abnormal mitochondrial morphology (swollen mitochondria with disorganized cristae), depletion of tyrosine hydroxylase positive neurons and dopamine levels [41]. Knockout of Drosophila pink1 has shown to produce similar morphological changes and climbing defects however with milder age-dependent dopaminergic cell death [42]. In regard to Parkin, the E3 domains of Drosophila parkin share 42\% amino acid sequence homology with those in human Parkin [43]. In knockout flies, age-dependent motor impairment and dopaminergic death are mild whereas male sterility and climbing defects associated with apoptotic muscle degeneration are prominent [43]. On the other hand, knockdown of parkin has been shown to result in age-dependent motor impairment alongside severe dopaminergic neuron loss. Additionally, non-motor features such as impaired memory and disturbed circadian rhythms have been noted upon knockdown of pink1/parkin $[19,44]$. The slight variations in phenotypes between knockdown/knockout models are currently attributed to complex mechanisms of genetic compensation. Such differences are not exclusive to flies and have been observed across most animal models of recessive PD [19, 24]. Overall, pink1/parkin flies stand as promising models to study the pathogenesis of PD as mutant flies display multiple phenotypes in addition to dopaminergic death thus strongly reflecting the multi-faceted nature of PD.

PINK1 and Parkin are implicated in a variety of fundamental cellular processes that are crucial influencers of dopaminergic neuron survival [45]. The following sections will attempt to explain the dysregulation of these mechanisms in PD and expand on the insights gained from Drosophila models of PINK1/Parkin.

\section{Mitochondrial dynamics in PD}

The earliest evidence that linked mitochondrial dysfunction to nigrostriatal death came from patients who abused a drug containing the neurotoxin MPTP (1-methyl-4-phenyl-1,2,3,6-tetrahydropyridine) [46]. MPTP is one among many neurotoxins known to cause progressive dopaminergic loss by disturbing mitochondrial respiration, ROS metabolism and fission/fusion dynamics within SNpc neurons $[47,48]$. Analysis of cells derived from patients with Parkin, PINK1 and LRRK2 mutations have noted patterns of abnormal mitochondrial morphology indicative of mechanisms driven by fission/fusion, lysosomal and trafficking proteins [4951]. These observations have led to extensive transgenic animal studies looking into the aberrant interactions between familial genes and proteins involved in mitochondrial dynamics [52].

Mitochondria are highly intricate organelles which serve as the fundamental source of energy within neurons [53]. The double membraned organelles contain respiratory complexes distributed throughout their highly folded cristae which synthesize adenosine triphosphate (ATP) via oxidative phosphorylation. Both membranes harbour GTPases that circumvent the fission and fusion of mitochondria based on the requirements of the neuron [54]. Mitofusins (Mfn1, Mfn2) facilitate the fusion of outer mitochondrial membranes (OMM) while optic atrophy 1 (OPA1) mediates inner membrane (IMM) fusion. Conversely, dynamin related protein (Drp1), mitochondrial fission-1 protein (Fis1) as well as certain adaptor proteins localize at mitochondria-endoplasmic reticulum (ER) contact sites to facilitate the division of mitochondria into smaller fragments [55]. These smaller mitochondrial fragments are of higher motility which allow for increased localization near nerve endings where synaptic transmission occurs [56, 57]. Fusion of mitochondria that have healthy intact genomes with those that are acutely damaged may also help to prolong the functionality of the latter [58]. Aside from maximizing the availability of ATP for energy driven processes such as neurotransmission via the aforementioned mechanisms, mitochondria undergo vicious cycles of fusion and fission to prime damaged mitochondria that are beyond repair for degradation [59].

Dysfunctional mitochondrial dynamics are a key source of metabolic stress within neurons. The following sections will detail the behaviour of the PINK1/Parkin axis and how it affects mitochondrial pathology within the context of PD.

\section{PINK1/Parkin regulation of fusion and fission}

In the event of mitochondrial damage due to assault by reactive oxygen species, severe membrane depolarization or accumulation of matrix debris, Parkin endorses sequestration of malfunctioning mitochondria by inhibiting mitochondrial fusion and fission while promoting mitophagy [60, 61]. In mammalian HeLa cells, Parkin ubiquitinates mitochondrial fusion proteins mitofusins 1 and 2 (Mfn1 and Mfn2) marking them for p97 (ATPase) assisted proteasomal degradation to prevent fusion and prepare for mitophagy [62]. Knockdown of Marf (Drosophila ortholog of human $M f n 2$ ) was shown to reduce abnormal mitochondrial morphology in the muscles of pink1 and parkin mutants thus confirming the mitoprotective effects of parkin mediated inhibition of fusion [63]. As stated earlier, the dynamin related protein Drp1 is a cytosolic GTPase that functions as a mitochondrial 
fission protein which surrounds and constricts mitochondrial membranes resulting in division (reaction powered by GTP hydrolysis) [64]. In mammalian cells, Parkin localizes on impaired mitochondria and ubiquitylates Drp1 priming it for proteasomal degradation hence rescuing cells from the accumulation of damaged mitochondria by inhibiting mitochondrial fission [65]. Conversely, studies involving Drosophila parkin and pink1 mutants have revealed that parkin overexpression in flies promotes mitochondrial fission through Drp1 and Fis1. Overexpression of the Drp1 ortholog in flies rescued abnormal wing posture and suppressed muscle degeneration in pink1 and parkin mutants [66]. The divergence in pathways suggests that there may be distinct factors that regulate Drp1 mediated mitochondrial fission in addition to the pink1/parkin axis in flies. Interestingly, two mechanisms have been elucidated regarding the latter, one being via autophagy related gene 1 (Atg-1) and the other through four-wheel drive (Fwd) kinase $[67,68]$.

\section{Atg-1 and Fwd target Drp1-mediated fission}

In addition to being involved in the turnover of dysfunctional organelles via autophagy, Atg-1 overexpression in pink1/parkin mutant flies has been shown to promote the sequestration of healthy mitochondria via upregulation of Drp1 mediated fission. Following Atg-1 knockdown in pink1 mutant flies, flight muscles were consistent with large irregularly shaped mitochondria which appeared to be as a consequence of abrogated fission and uninhibited fusion. Subsequent overexpression of Drp1 rescued the aforementioned phenotypes and prolonged dopaminergic neuron survival in fly brains. Furthermore, Atg-1 regulated fission was attributed to an increase in post transcriptional modification of Drp1, enhancing its activity and therefore fission. It has been suggested that since mitochondrial dynamics and Atg-1 expression are both nutrient driven, likewise is the induction of Atg-1 mediated fusion [67]. Another pathway that has garnered similar interest involves four-wheel drive (Fwd) kinase (the Drosophila ortholog of phosphatidylinositol 4-kinase III- $\beta$ ), a phosphoinositide that regulates membrane trafficking within cells. Analogous to pink1 null phenotypes, loss of four-wheel drive $(F w d)$ has been shown to trigger hyperfusion of mitochondria, locomotor defects and reduce the lifespan of flies. Surprisingly, while overexpression of Drp1 could rescue pink1/parkin null flies, it failed to rescue pink1/parkin/Fwd (triple mutant) phenotypes thus inferring that Drp1-mediated fission may be dependent on Fwd expression [68]. Currently there lies no detailed explanation on the molecular interactions between these proteins and so before these pathways are put forth as therapeutic targets, the specific conditions which are needed to evoke them and their behaviour in mammalian models require further investigation.

\section{MUL1 targets mitofusins alongside Parkin to inhibit fusion}

With regard to fusion dynamics, the mitochondrial ubiquitin ligase (MUL1) has been found to target mitofusins in a similar manner to Parkin mediated ubiquitylation and degradation following mitochondrial stress [69]. MUL1 is a small ubiquitin-like modifier (SUMO) E3 ligase localized to the OMM which has been reported to SUMOylate Drp1 and prime cells for apoptosis [70]. According to a recent study, overexpression of the MUL1 ortholog in flies (Mul1) has been shown to suppress phenotypes caused by overexpression of mitofusins, knockdown of pink1 or parkin. The study hypothesized that Mul1 operates in parallel to pink1 and parkin as all double mutants (pink1/Mul1, parkin/Mul1) showed exacerbated phenotypes compared to single mutants. Moreover, the functional importance of Mul1 in inhibiting mitochondrial fusion was demonstrated when pink1/ parkin double mutants showed milder phenotypes than pink1/Mul1 and parkin/Mul1 double mutants. MUL1 mediated ubiquitylation of mitofusins was also confirmed in mammalian cells [69]. While it has been suggested that upregulation of MUL1 might serve as a novel method to target mitochondrial dysfunction in PD, a certain polymorphism in the MUL1 gene was recently identified as a risk factor for PD in a cohort of Chinese patients [71]. Further investigation into the downstream effects of this variant could bring new insights into the importance of MUL1 in cellular dynamics and if it possibly contributes to PD pathology in a way that broadens our current understanding.

These studies make clear that mitochondrial fission and fusion are highly complex processes and further study into additional influencing factors would provide greater insight into the role of fission/fusion dynamics in neural health.

\section{PINK1/Parkin regulation of mitophagy}

As earlier stated, the clearance of dysfunctional mitochondria via mitophagy is a critical process in neurons. Apart from evoking UPS mediated degradation, Parkin may target outer mitochondrial proteins (OMP) such as VDAC1 (voltage-dependent anion channel 1) or mitofusins (Mfn2) to induce mitophagy [60, 61]. Ubiquitination and phosphorylation of such membrane proteins recruits autophagic receptors such as NDP52, Tax1-binding protein 1 (TAXBP1) or Optineurin (OPTN) which bind to phosphor-ubiquitin chains on the ubiquitylated cargo while interacting with phagophore membranes 
(LC3/Atg8) to form a mitophagosome which then undergoes lysosomal degradation [72].

PINK1/Parkin mediated mitophagy is regulated by deubiquitinating enzymes such as USP8, USP15 and USP30. Self-ubiquitination of Parkin (K6 linked) is a form of autoinhibition that restrains Parkin from proteasomal degradation and mitochondrial recruitment. USP8 severs K6 linkages on ubiquitylated Parkin to endorse mitophagy following stress-induced depolarization of the OMM [73]. In Drosophila studies, silencing of CG8334 (Drosophila homolog close to human USP15), was observed to improve locomotor ability and reduce mitochondrial clustering in parkin RNAi flies. USP15 was found to deubiquitinate K48 and K63 linked OMP thus antagonizing parkin mediated mitophagy [74]. Additionally, knockdown of CG3016 (Drosophila homolog of human USP30) reduced the percentage of swollen mitochondria with disorganized cristae in parkin mutant flies [75]. USP30 was found to severe K6 linkages on TOM20, Miro1 and parkin itself. Thereby blocking parkin mediated mitophagy while also promoting the proteasomal degradation of parkin [76-78]. Thus, inhibition of USP15 and USP30 activity could serve as a therapeutic approach to ameliorate PD phenotypes that are aggravated by defective mitochondrial clearance [79].

\section{PINK1/Parkin induce mitophagy via Miro GTPase}

Considering that dopaminergic neurons have slender axons and are of limited myelination compared to other classes of neurons within the substantia nigra, the compounding effects of defective mitochondrial clearance, fission/fusion dynamics and mitochondrial transport can be deleterious to their health [80]. Anterograde transport of mitochondria towards the synaptic terminals is associated with kinesin motor proteins while retrograde transport to the soma is facilitated by dynein motors [81]. A class of mitochondrial Rho GTPases bound to the OMM known as Miro proteins interact with the kinesin/dynein motors via the adaptor protein Milton/ TRAK (Trafficking kinesin-binding protein) to regulate mitochondrial transport along axons [82]. According to Drosophila studies, pink1 mediated phosphorylation (at Ser182/324 or Thr325) and parkin mediated degradation of Miro forces the detachment of damaged mitochondria from the motor protein complex, possibly in preparation for mitophagy [83, 84]. Expression of unphosphorylated Miro in pink1 inactivated flies has been shown to increase axonal mitochondrial movement, synaptic overgrowth and reduce the number of dopaminergic neuron clusters amongst fly larval motor neurons [83]. While Drosophila pink1 mutants show upregulated levels of Miro and eventually suffer from significant dopaminergic loss, PINK1/Parkin inactivated murine models do not [84]. The difference in phenotypes does not necessarily imply that there are no pathological consequences of this process in humans. In fact, impeded degradation of Miro in fibroblast cells derived from PD patients with PINK1, Parkin and LRRK2 mutations has confirmed the clinical importance of mitochondrial motility and impaired mitophagy in PD [51, 85]. Thus, the difference in phenotypes between flies and rodents could be attributed to different compensatory mechanisms that exclude the PINK1/Parkin axis. There are differing theories on the interaction between PINK1/Parkin and Miro in humans. Studies in HeLa cells initially demonstrated that PINK1 requires the co-expression of Parkin to downregulate Miro levels and clear defective mitochondria via mitophagy [86]. Conversely, more recent studies have suggested that degradation of Miro can occur independently of PINK1 phosphorylation and that Parkin interacts with Miro prior to mitochondrial depolarization in the event of neural calcium overload. The latter is hypothesized to induce Parkin mediated polyubiquitination (K572 linkage) of Miro followed by mitophagy. Furthermore, this pathway has been reported to unfold alongside the PINK1/Parkin pathway that ensues following stress-induced depolarization [87]. While the link between impaired regulation of Miro and neurodegeneration in recessive PD warrants further investigation, current studies present promising evidence that dysfunction of Miro is a contributing factor [88].

Overall, these studies provide different explanations for the pattern of mitochondrial aggregation within dopaminergic neurons and underscore the significance of dysfunctional mitochondrial dynamics in recessive PD.

\section{Oxidative stress in PD}

The stability of ETS (electron transport system) complexes is critical in neurons. When mitochondrial respiration is compromised, it leads to the depletion of ATP which has severe implications for the synaptic activity of neurons (from affecting the generation of action potentials to neurotransmitter release) [89, 90]. Impaired ETS activity along with the deficiency of antioxidant enzymes could also accelerate the accumulation of reactive oxygen species (ROS) thus propagating dopaminergic neuron loss [91]. Clear evidence of oxidative damage in PD brains has been made following the detection of 8-hydroxyguanine (oxidatively modified nucleic acid) and 4-hydroxynonenal (product of lipid peroxidation) within neurons of the substantia nigra $[92,93]$. Complex 1 of the ETS has also been shown to undergo oxidative modification of its subunits to present protein carbonyl residues that correlate with decreased complex I activity [94]. In terms of ETS induced oxidative stress, several studies have disputed whether the instability of ETS complexes 
arises from mutations in mitochondrial DNA, as a result of exposure to environmental toxins (paraquat, rotenone) or both $[95,96]$. The main mechanism of ROS accumulation via the ETS appears to occur through the leakage of electrons from dysfunctional complexes [97].

\section{ETS complexes lead the ROS cascade}

From the mitochondrial matrix, intermediates of the tricarboxylic acid cycle (TCA cycle) NADH (reduced nicotinamide adenine dinucleotide) and FADH2 (reduced flavin Adenine dinucleotide) are reduced upon donating electrons to complex I and complex II in the IMM. The shuttling of electrons from complex I and II to complex III (via coenzyme Q) and subsequently complex IV (via cytochrome c) induces a proton gradient across the IMM (chemiosmosis) that drives the synthesis of ATP from ADP and Pi (via ATP synthase); a process known as oxidative phosphorylation [54]. Electrons that leak from complex I and complex III are accepted by oxygen in the matrix to form superoxide anions. These superoxide anions are converted to hydrogen peroxide by the antioxidant enzyme superoxide dismutase (SOD). Lack of superoxide clearance can affect the stability of proteins such as aconitase (Krebs cycle enzyme) and complex I itself. Superoxide targets iron-sulphur clusters within these proteins resulting in their inactivation and the release of $\mathrm{Fe} 2+[97,98]$. Hydrogen peroxide reacts with the latter to produce hydroxyl radicals (Fenton and Haber-Weiss reactions) which precipitate the oxidative modification of nucleic acids (causing mitochondrial DNA damage), lipid peroxidation (affecting mitochondrial membrane stability) and protein damage (modification of ETS complexes) [99]. ROS may also promote apoptosis by inducing the release of cytochrome $\mathrm{c}$ through the oxidation of cardiolipin, or by interacting with MPTP proteins [97-99].

\section{PINK1/Trap1 protect against oxidative stress by regulating complex I activity}

According to Drosophila studies, pink1 mutants have been shown to exhibit impaired synaptic transmission and increased sensitivity to ROS assault as a consequence of complex I inactivity [100]. While PINK1 does not directly participate in redox homeostasis through regulation of ETS complexes, PINK1 mediated phosphorylation of the mitochondrial chaperone tumor necrosis factor (TNF) receptor-associated protein 1 (Trap1) has been observed to exert protection against ROS induced stress. Upon phosphorylation, Trap1 and PINK1 become colocalized to the IMM as well as the intermembrane space [101]. However, it is important to note that other fly studies have shown that Trap 1 activity may not necessarily be entirely dependent on PINK1 phospho-activation to exert mito-protection. In discussion of its mito-protective role, it has been established that certain heat shock protein (Hsp60/70/100) classes are highly associated with mitochondrial biogenesis by regulating the assembly, folding and translocation of various mitochondrial proteins [102]. Being a member of the Hsp90 (heat shock protein 90) family of chaperone proteins, it is possible that Trap1 could affect the folding and assembly of respiratory subunits. In support of this theory, ablation of the Trap1 ortholog (Trap1) in pink1 mutant flies has been shown to lower complex I expression and ATP levels eventually culminating in dopamine deficiency. These defects were then rescued following ectopic Trap1 expression. In addition, the latter improved the locomotor ability and survival of pink1 mutants that were subjected to paraquat induced neurotoxicity [103]. These results thus indicate that Trap1 may be key in inhibiting the onset of ROS build up via the ETS as well as strengthening the organellar response following ROS assault.

\section{Sir2 and Trap1 inhibit oxidative stress through FOXO}

Interestingly, recent studies have proposed an alternative mechanism for the influence of Trap1 on oxidative stress. According to one study, enhanced expression of Drosophila Trap1 failed to rescue ROS-induced mitochondrial defects expressed by pink1 mutant flies. Instead, the phenotypes were ameliorated following the knockdown of Trap1. The study went on to report that suppression of Trap1 resulted in increased expression of the transcriptional activator forkhead box O (FOXO) [104]. FOXO expression has been known to impede ROS build up by upregulating transcription of genes encoding the antioxidant enzyme superoxide dismutase 2 (SOD2). Interestingly FOXO factors have been found to operate in a feedback loop triggered by the accumulation of matrix proteins which accelerate ROS production to in turn activate FOXO factors [105]. Although this particular feedback mechanism has not been studied in flies or mammalian models, it presents FOXO as an interesting hypothetical target for exploitation in PD. In addition to Trap1, expression of FOXO has also been shown to be dependent on pink1. Through an elusive mechanism, pink1 has been hypothesized to activate the histone deacetylase silent information regulator 2 (Sir2) which deacetylates FOXO to trigger transcription of target genes (SOD2, 4E-binding protein). Ectopic expression of Sir2 and FOXO in pink1 null flies was observed to recover functional wing posture, flight muscle activity, mitochondrial morphology, ATP levels and reduce dopaminergic neuron loss [106]. Taken together, these mechanisms paint a complex picture of how Trap1 may mediate the response to oxidative stress. 


\section{UCP4 works against the ETS to ameliorate oxidative stress}

While the ETS remains a huge source for ROS build up, additional IMM proteins also partake in ROS metabolism, one being a member of the family of uncoupling proteins. Uncoupling protein 4 (UCP4) belongs to a family of mitochondrial solute carriers (SLC25) which include the homologues UCP1-5. UCP4 serves as an anion channel embedded in the IMM which facilitates the leakage of protons from the intermembrane space back into the matrix, by-passing complex V (ATP synthase) mediated proton transfer during oxidative phosphorylation. Aside from reducing the potential of a highly polarized mitochondrial membrane, the resulting proton pool in the matrix scavenges any free radicals to truncate the ROS cascade and does so without affecting ATP production. It has been hypothesized that UCPs function in a negative feedback loop with UCPs being modified into their active state by 4-hydroxyhexanal, a product of lipid peroxidation that is produced downstream of the ROS cascade. This way UCPs inhibit membrane hyperpolarization and ROS build-up [107, 108]. In addition to regulating oxidative stress, UCP4 expression has been associated with the uptake of succinate into the mitochondria to drive complex II activity and oxidative phosphorylation [109]. Overexpression of the UCP4 ortholog in flies (UCP4A) has been shown to suppress respiratory defects (rescue low complex I and ATP levels), flight muscle degeneration and mortality from paraquat or rotenone induced oxidative stress in pink1 mutants. UCP4A overexpression also ameliorated similar motor defects in parkin flies. While these studies ascertained that UCP4A functions downstream of pink1 and parkin, the exact relationship between these proteins remains to be elucidated [78]. Interestingly, the link between recessive PD and UCP4 has been further established with rodent studies showing that mutant forms of $D J-1$ (PARK7) result in lower UCP4 expression via the NF- $\mathrm{kB}$ (Nuclear factor kappa B) pathway therefore propagating neural death [111]. The regulation of $\mathrm{UCP} 4$ within the confines of PD is therefore rather complicated and further investigation should clarify its therapeutic potential in recessive PD.

\section{PGC1a expression ameliorates ROS stress via the PINK1/Parkin axis}

Peroxisome proliferator-activated receptor gamma coactivator-1- $\alpha(\mathrm{PGC} 1 \alpha)$ is a transcriptional coactivator known to modulate a variety of cellular processes such as glucose metabolism, fatty acid oxidation, thermogenesis and organellar metabolism. PGC1 $\alpha$ primarily exerts its mito-protective role by interacting with specific transcription factors such as transcription factor
A, mitochondrial (TFAM) and nuclear respiratory factor-1 (NRF-1). Through such factors, PGC1 $\alpha$ regulates mitochondrial respiratory and oxidative capacity by upregulating the synthesis of TCA cycle enzymes, the expression of respiratory complexes (ETS) and mitochondrial antioxidant enzymes (superoxide dismutase and glutathione peroxidase) [112]. From recent studies, the $\mathrm{KRAB} /$ zinc finger binding protein PARIS(ZNF746) has been reported to target the promoter sequence of PGC1 $\alpha$ resulting in its suppression and further precipitation of nigral dopaminergic death [113]. Phosphorylation of PARIS (at Ser322/613) by PINK1 and ubiquitination by Parkin triggers its degradation thus allowing PGC1 $\alpha$ to exert its mito-protective effects on neurons [114]. In Drosophila pink1/parkin mutant flies, overexpression of the PARIS ortholog (dPARIS) was observed to result in age-dependent climbing defects, dopaminergic neuron loss, increased lethality as well reduction of mitochondrial DNA copy number. The aforementioned phenotypes were then rescued following knockdown of $d P A R I S$ thus highlighting its therapeutic potential [115]. Similar to PARIS-induced repression, the activity of PGC1 $\alpha$ has been reported to be affected by several other pre-/posttranscriptional modifications and so further research into additional regulatory factors could clarify the nature of this axis in PD and help define the correct approach for therapy [116].

\section{Wnt2 impedes oxidative stress via PGC1a and FOXO}

There is a growing body of research being dedicated to the application of canonical Wnt (Wingless-type mouse mammary tumor virus (MMTV) integration site) signalling in neurodegenerative disorders. From promoting proliferation and differentiation of dopaminergic neurons within the midbrain to axon/dendrite and synapse formation, Wnt signalling molecules are integral players in CNS health $[117,118]$. Canonical Wnt signalling involves the cell-surface receptor Frizzled (Fzd) and co-receptor LDL-receptor-related protein (LRP5/6). Binding of the Wnt ligand to these receptors results in the recruitment of the protein Dishevelled (Dvl) and a destruction complex to the plasma membrane. The complex is composed of casein kinase 1 (CK1Y), glycogen synthase kinase 3 (GSK3 $\beta$ ), axin and adenomatous polyposis coli (APC). CK1 $\gamma$ and GSK3 $\beta$ then phosphorylate LRP tails which sequester axin. This allows $\beta$-catenin to translocate to the nucleus, displace Groucho (co-repressor) and bind to transcription factor-like T-cell factor and lymphoid enhancer-binding factor (TCF/LEF) thus inducing transcription of Wnt genes [119]. Recent studies in Drosophila have shown Wnt2 overexpression in pink1 mutant flies to rescue flight muscle degeneration 
and neural activity. Overexpression of Wnt2 particularly improved ATP levels, mitochondrial morphology, mRNA expression of $\mathrm{NADH}$-ubiquinone oxidoreductase chain 1 (ND1), cytochrome b and succinate dehydrogenase complex subunits. ROS production was also seemingly downregulated following the detection of reduced levels of malondialdehyde (a product of lipid peroxidation) and increased levels of manganese superoxide dismutase (MnSOD). Here, the protection against oxidative stress by Wnt 2 was attributed to enhanced PGC1 $\alpha$ and FOXO expression [120]. While details of the molecular relation between PGC1 $\alpha$ and Wnt expression for neural protection are unclear, previous studies have suggested that ROS build-up endorses the binding of $\beta$-catenin to FOXO thereby enhancing its transcriptional activity [121]. Moreover, although this axis provides an interesting perspective to the contribution of Wnt in PD progression and its possible therapeutic application, previous studies have related Parkin mutations to the dysregulation of the canonical Wnt axis. Parkin mutations have been shown to cause aberrant Wnt signalling resulting in the build-up of cyclin $E$ and re-entry of differentiated neurons into the cell cycle which eventually led to their death [122].

ROS stress is a prime cause for the acceleration of dopaminergic death. Going forward, further research into the interplay between the PINK1/Parkin axis and the aforementioned signalling pathways could help uncover the existence of additional protective mechanisms while also clarifying the elusive details of those mentioned above.

\section{Protein misfolding in PD}

Another important factor that has been alluded to escalate neural death stems from the accumulation of toxic proteins [123]. The assembly of proteins into fully functional oligomeric complexes is a highly sophisticated process. Mutations in the gene that encodes the particular protein or in genes that encode folding-machinery coupled with exogenous assault by neurotoxic agents can all precipitate protein misfolding. To a certain extent protein misfolding is inevitable and there are different clearance systems designed to prevent their accumulation. For instance, the latter may be effectively degraded via the UPS, the autophagy-lysosomal pathway or may be remodelled into their correct conformations via chaperone proteins [124]. The stress induced by such protein aggregates can disrupt cellular metabolism at any level. More specifically, the toxic cellular effects have been described as mutually exacerbating in that protein aggregates can initiate a disruption of metabolism (such as $\mathrm{ROS} / \mathrm{Ca} 2+$ homeostasis) that may in return accelerate pathology (such as neuroinflammation) induced by the former [125]. While the lack of clearance of any misfolded protein is bound to perturb the health of neurons, the accumulation of misfolded $\alpha$-synuclein has been characterized as a hallmark indicator of sporadic and dominantly inherited forms of PD. $\alpha$-synuclein aggregates to form Lewy body inclusions throughout the brain and has been hypothesized to induce toxicity by affecting mitochondrial function, proteasomal clearance systems, membrane stability, and dopamine transport within infiltrated neurons [126]. With respect to recessive PD, several Parkin substrates such as CDCrel-1 (synaptic vesicle associated GTPase), Pael-R (ER localized GPCR) and Synphilin-1 have also been associated with neurotoxicity following their accumulation [123].

\section{Parkin regulates the clearance of misfolded proteins via aggresomes}

Aside from UPS mediated degradation, Parkin has been shown to promote the formation of aggresomes (clumps of misfolded proteins) [127]. This method of protein clearance has been reported to unfold when traditional UPS and chaperone systems are overwhelmed. The E2 enzyme UbcH13/Uev1a and Parkin interact to polyubiquitylate misfolded proteins (K63 linkage) which then bind to the adaptor protein histone deacetylase 6 (HDAC6) and the dynein motor complex to form an aggresome. The aggresome is then sequestered into an autophagosome and degraded [128]. Although the exact fate of substrates under K63 linkage varies, Parkin has been shown to adopt this method to target misfolded DJ-1 and synphilin-1. Synphilin-1, a protein that is associated with $\alpha$-synuclein and Lewy body formation, is a peculiar target as Parkin mutants are not typically associated with Lewy body pathology [127-129]. This provides an interesting line of research to further probe the possible link between the aforementioned proteins and their relevance in PD pathology.

\section{CHIP overexpression rescues Parkin mutants from mitochondrial and ER stress}

Accumulation of misfolded proteins within the endoplasmic reticulum (ER) has also been proposed as a potential cause for dopaminergic cell death. When misfolded/ unfolded proteins accumulate it sets off a response known as the unfolded protein response (UPR) which involves the activation of a series of signal transduction pathways to cope with ER-stress. The signals may culminate in ER-assisted degradation (ERAD), wherein misfolded proteins are translocated out of the ER into the cytosol for ubiquitination and degradation [130]. As mentioned earlier, Pael-R, a G protein-coupled transmembrane receptor, is a Parkin substrate that is synthesized within the ER. Accumulation of unfolded Pael-R has been shown to exert ER-stress and neurotoxicity in 
patients with Parkin mutations [131]. Studies in neuronal cell lines have elucidated that the carboxyl terminus of Hsc70-interacting protein (CHIP) interacts with Parkin to determine the fate of misfolded proteins. Through its U-box domain, CHIP acts as an E3 ubiquitin ligase on interaction with other heat shock proteins ( Hsp90, Hsp70) to mitigate protein folding and clearance. CHIP has been found to prime misfolded/unfolded proteins for degradation via proteasomes or aggresomes in a chaperone dependent or independent manner [132]. Importantly, CHIP has been shown to co-localize with Parkin at the surface of the endoplasmic reticulum to sequester unfolded Pael-R from Hsp70 and cochaperone Hdj-2 for subsequent ubiquitylation and degradation. In addition to enhancing Parkin ligase activity, CHIP was also found to interact with other E2 enzymes such as Ubc4/6/7 to ubiquitylate unfolded Pael-R [133].

While these studies established that CHIP acts to alleviate ER-stress via Parkin, studies in flies have revealed that overexpression of Drosophila CHIP can rescue mitochondrial dysfunction in pink1 and parkin mutants [134]. More specifically, CHIP was found to positively affect the climbing ability and thoracic ATP levels of flies while also suppressing dopaminergic neuron loss. Overexpression of CHIP in fly models rescued parkin mutant phenotypes while parkin overexpression was required to assist CHIP in rescuing pink1 mutants. This inferred that CHIP functions downstream of pink 1 and in parallel with parkin [134]. However, the molecular mechanisms behind these results require further clarification. There are few studies that suggest that mitochondrial dysfunction as a result of the accumulation of misfolded mitochondrial proteins confers dopaminergic neuron death. Being a molecular chaperone, whether CHIP aids in the clearance of misfolded respiratory complexes, mitochondrial fission and fusion proteins, or matrix proteins is currently unknown [102]. Moreover, the specific characteristics of the association between CHIP, PINK1 and Parkin are yet to be elucidated. Thus, these factors need to be taken into consideration before CHIP is exploited for PD therapy.

Protein toxicity is a poorly understood cause of neurodegeneration in PD. Thus, further research into additional defective clearance mechanisms such as those described above is needed to broaden our understanding of its role in PD pathogenesis.

\section{Apoptosis in PD}

From aberrant mitochondrial dynamics to protein misfolding and oxidative stress, the cellular triggers for dopaminergic death are plenty [135]. Initial evidence of the correlation between apoptotic death and PD stemmed from histological analysis of SNpc tissue taken from postmortem PD brains. With SNpc dopaminergic neurons showing heightened DNA fragmentation, chromatin condensation, cell body (soma) shrinkage and formation of apoptotic bodies, it seemed evident that apoptosis was most likely a key feature in PD pathogenesis [136, 137]. In addition to morphological markers, elevated expression of Bcl-2 (B-cell lymphoma 2) pro-apoptotic proteins, caspase-8, caspase- 9 and executioner caspase- 3 have been detected in post-mortem and in vivo studies [138-140]. While there are reports of the extrinsic (death receptor mediated) pathway of apoptosis being implicated in $\alpha$-synuclein associated neuroinflammation, the intrinsic (mitochondrial) cascade is widely considered to be the predominant pathway of cell death in PD [141]. As shown by neurotoxic models (MPTP, Rotenone and 6-hydroxydopamine), impaired complex I activity and complex I mediated oxidative stress are largely responsible for the activation of pro-apoptotic proteins (Bcl-associated $\mathrm{X}$ protein (Bax)) and inducing cytochrome c release [142144]. Furthermore, dopaminergic neurons have been reported to be increasingly sensitive to ROS-induced apoptosis due to the oxidative deamination of dopamine into hydrogen peroxide by the enzyme monoamine oxidase B (MAOB) [145]. The autocatalytic production of this radical compounded with insult via the aforementioned stressors, sets up a highly pro-apoptotic environment culminating in dopaminergic death.

With regard to recessive PD, several studies have identified apoptotic regulators Bcl-xL (B-cell lymphomaextra-large), Bax (Bcl-associated $\mathrm{X}$ protein) and Bak (Bcl-1 antagonist/killer-1) to interact with PINK1 and Parkin $[146,147]$. These proteins are key players in what is known as the intrinsic/mitochondrial pathway of programmed cell death. The pathway is triggered by the release of cytochrome $\mathrm{c}$ from the intermembrane space into the cytosol via $\mathrm{Bcl}-2$ pro-apoptotic proteins $\mathrm{Bax}$ and Bak [148]. Bax and Bak interact with other Bcl-2 family proteins via $\mathrm{BH} 3$ domains/grooves. $\mathrm{BH} 3$ grooves of Bak/Bax form symmetric dimers and subsequent homooligomers through their transmembrane $\alpha 5 / \alpha 6$ domains [149]. Oligomerized Bak/Bak molecules interact with the mitochondrial apoptosis-induced channel (MAC) which permeabilizes the mitochondrial outer membrane to release cytochrome c. Cytochrome $\mathrm{c}$ then binds to the adaptor protein Apaf1 (apoptotic protease activating factor 1 ) causing it to oligomerize and activate a caspase mediated cascade (initiator caspase 9 and executioner caspase 3) culminating in caspase 3 mediated DNA fragmentation, membrane blebbing and cell death [150]. Apoptosis is primarily regulated through interactions between Bcl-2 family members. Pro-apoptotic BH3only protein Bid (BH3-interacting domain death agonist) is truncated into its active state tBid to trigger Bak oligomerization (activation) by inducing conformational 
changes in Bak (displacement of N-terminus or C-terminus and BH3 domain exposure) [151]. Pro-survival Bcl-2 forms hetero-dimers with Bak to block oligomerization and thence apoptosis. In response, pro-apoptotic $\mathrm{BH} 3-$ only protein Bim (Bcl-2 interacting mediator of cell death) may indirectly trigger apoptosis by displacing Bak bound to anti-apoptotic Bcl-2 [152].

\section{PINK1/Parkin modulate the intrinsic apoptotic pathway}

PINK1 promotes apoptosis following stress-induced mitochondrial membrane depolarization by phosphorylating a serine residue (Ser 62 ) on the Bcl-xL domain which would otherwise undergo depolarization induced cleavage (at Asp 61), rendering it inactive [147]. Conversely, Parkin has been shown to ubiquitylate Bak (K11 linkage) inhibiting its homo-dimerization, oligomerization and subsequent cytochrome c release thereby rescuing cells under acute mitochondrial stress from apoptosis [146]. Interestingly, parkin regulated calcium efflux via mono-ubiquitinated VDAC1 has been identified as an alternative pathway in which parkin upregulates apoptosis. Studies in Drosophila have demonstrated that parkin mutants with defective porin (Drosophila ortholog of $V D A C 1)$ exhibit increased locomotor defects and apoptosis alongside characteristic PD-phenotypes. VDAC1 is an OMM channel protein that increases mitochondrial membrane permeability towards cytochrome $\mathrm{c}$ via association with adenine nucleotide translocator (ANT) and cyclophilin D (together forming the mitochondrial permeability transition pore/MPTP). Bcl-2 interacts with the $\mathrm{N}$-terminus region of VDAC1 directly blocking cytochrome c release while Bax forms a hetero-oligomeric complex with VDAC1 to facilitate cytochrome c release [153]. Further analysis in Drosophila revealed that while parkin mediated monoubiquitination of VDAC1 (K274 linkage) led to inhibition of apoptosis, polyubiquitination of VDAC1 (K12/20/53/109/110 linkage) promoted mitophagy. As predicted, monoubiquitinated VDAC1 failed to attract Bax thus suppressing apoptosis. In $V D A C 1$ K274R flies (defective in monoubiquitination), mitochondrial swelling as a result of excess calcium influx increased apoptosis. Subsequent knockdown of mitochondrial calcium uniporter (MCU) rescued VDAC1 $K 274 R$ phenotypes suggesting that VDAC1 regulation of calcium influx was key to inducing apoptosis [154]. The specific cellular conditions that selectively induce parkinVDAC1 monoubiquitination over polyubiquitination are however unclear and further investigation into the associated mechanisms should clarify the link between parkin-VDAC1 assisted mitophagy and apoptosis in flies.

In summary, these mechanisms confirm that PINK1 and Parkin play multiple roles in influencing programmed cell death. However, a more detailed understanding of their interactions with other components of the apoptotic cascade is needed to advance therapeutic strategies.

\section{Screening for potentially therapeutic compounds in Drosophila melanogaster}

So far, treatment for PD has revolved around symptomatic strategies such as pharmacotherapy, rehabilitative medicine and in some cases surgery. Spearheaded by the administration of medications that act to upregulate dopamine levels (levodopa, dopamine agonists, COMT/ MAOB inhibitors), pharmacotherapy is often plagued by a wide array of side effects (dyskinesias) and overall fails to halt the progression of the disease. On the other end of the spectrum, rehabilitative methods take time and the guarantee of a successful prognostic outcome is difficult to predict [2]. Therefore, there is an ever-growing need to develop a line of therapy that brings about significant short-term improvement with minimal side effects.

Beyond identifying molecular rescues, fly models have also been used as a system for screening a multitude of possible neuroprotective compounds. Compared to murine models, flies allow for quick and cost-effective high throughput drug screening [155]. The earliest screens in fly models targeted locomotor dysfunction induced by $\alpha$-synuclein toxicity [156]. Geldanamycin, an antibiotic that promotes Hsp70 chaperone activity, was found to attenuate dopaminergic death and synuclein aggregation in SNCA mutant flies [157]. Screens have now expanded to target other aspects of PD pathogenesis such as oxidative stress and mitochondrial biogenesis (Table 1).

Several compounds have been shown to target ROS stress by increasing the transcription of antioxidant enzymes. Propyl gallate and epigallocathecin belong to a class of polyphenols that promote ROS clearance by inducing NAD-dependent deacetylase sirtuin-1 (SIRT1) activity [158]. Sirtuins (SIRT1/2) further activate transcription factors such as nuclear factor erythroid 2related factor 2 (Nrf2), PGC1 $\alpha$ and FOXO to induce the expression of antioxidant enzymes [159]. Supplementation of propyl gallate and epigallocathecin were shown to alleviate all climbing defects in parkin mutant flies [160]. Through similar mechanisms, sulforaphane, an isothiocyanate, was also shown to reduce dopaminergic neuron loss in parkin mutant flies [161]. Termed as the phase II detoxification pathway, sulforaphane prevented the sequestration of $\mathrm{Nrf} 2$ for proteasomal degradation by Kelch-like ECH associated protein 1 (KEAP1) and Cullin 3 (CUL3) thereby allowing Nrf2 to bind to antioxidant response elements (ARE) and transcribe for antioxidant enzymes (such as glutathione 
Table 1 Therapeutic compounds/extracts tested in Drosophila PINK1/Parkin models

\begin{tabular}{|c|c|c|c|c|}
\hline $\begin{array}{l}\text { Therapeutic compound/extract/ } \\
\text { protein }\end{array}$ & $\begin{array}{l}\text { Drosophila model } \\
\text { (gene knockdown/ } \\
\text { knockout) }\end{array}$ & Phenotype/s modified & Mechanism of action & References \\
\hline $\begin{array}{l}\text { Polyphenols: Propyl gallate, Epigal- } \\
\text { locathecin }\end{array}$ & parkin & $\begin{array}{l}\text { Improved lifespan and attenuated } \\
\text { climbing defects }\end{array}$ & $\begin{array}{l}\text { Inhibits ROS accumulation and } \\
\text { prevents iron induced neurotoxic- } \\
\text { ity }\end{array}$ & {$[158,160]$} \\
\hline Zinc chloride & parkin & $\begin{array}{l}\text { Improved lifespan and locomotor } \\
\text { ability }\end{array}$ & Impedes ROS-induced apoptosis & [165] \\
\hline Nicotine & parkin & $\begin{array}{l}\text { Improved lifespan, viability, loco- } \\
\text { motor ability and reduced olfactory } \\
\text { deficits }\end{array}$ & $\begin{array}{l}\text { Promotes activity of complex I and } \\
\text { reduces the production of ROS via } \\
\text { the ETS }\end{array}$ & {$[168,179]$} \\
\hline Minocycline & parkin & $\begin{array}{l}\text { Improved lifespan and locomotor } \\
\text { ability }\end{array}$ & $\begin{array}{l}\text { Protects against iron and paraquat } \\
\text { induced toxicity and scavenges } \\
\text { ROS }\end{array}$ & [166] \\
\hline Folic acid & parkin & $\begin{array}{l}\text { Improved lifespan and locomotor } \\
\text { ability }\end{array}$ & $\begin{array}{l}\text { Downregulates p53, PGC1 } 1 \text { and } \\
\text { ATP levels (promotes mitochon- } \\
\text { drial biogenesis and ROS clear- } \\
\text { ance) }\end{array}$ & [163] \\
\hline Sulforaphane & parkin & $\begin{array}{l}\text { Suppressed dopaminergic neuron } \\
\text { loss }\end{array}$ & $\begin{array}{l}\text { Stimulates Nrf2 induced transcrip- } \\
\text { tion of antioxidant enzymes }\end{array}$ & {$[161,162]$} \\
\hline $\begin{array}{l}\text { Resveratrol /Grape skin extract } \\
\text { (GSE) }\end{array}$ & pink1 & $\begin{array}{l}\text { Improved lifespan, wing posture, } \\
\text { locomotor ability and larval mito- } \\
\text { chondrial morphology }\end{array}$ & $\begin{array}{l}\text { Upregulates p62 receptor expres- } \\
\text { sion and mitophagy }\end{array}$ & [170] \\
\hline Ginseng protein & pink1 & $\begin{array}{l}\text { Improved wing posture, locomotor } \\
\text { ability and suppressed dopaminer- } \\
\text { gic neuron loss }\end{array}$ & $\begin{array}{l}\text { Triggers unfolded protein response } \\
\text { (promotes mitochondrial biogene- } \\
\text { sis) and protects against rotenone/ } \\
\text { paraquat induced toxicity }\end{array}$ & [171] \\
\hline Mucuna pruriens & pink1 & Improved SOD and GSH levels & $\begin{array}{l}\text { Increases clearance of ROS by } \\
\text { upregulating antioxidant enzyme } \\
\text { activity }\end{array}$ & [167] \\
\hline Recombinant SOD1 + Vitamin E & pink1 & $\begin{array}{l}\text { Reduced ommatidial and retinal } \\
\text { degeneration and suppressed } \\
\text { dopaminergic neuron loss }\end{array}$ & $\begin{array}{l}\text { Protects against ROS-induced cell } \\
\text { death (scavenges ROS) }\end{array}$ & [180] \\
\hline Vitamin $\mathrm{K}_{2}$ & pink1 & $\begin{array}{l}\text { Improved locomotor ability and } \\
\text { larval mitochondrial morphology }\end{array}$ & $\begin{array}{l}\text { Acts as an ETS carrier analogous to } \\
\text { ubiquinone and thereby promotes } \\
\text { oxidative phosphorylation }\end{array}$ & [169] \\
\hline
\end{tabular}

peroxidase, thioredoxin and heme-oxygenase 1) [162]. Folic acid is another therapeutic compound reported to influence ROS stress via PGC1 $\alpha$ activity. By inhibiting p53 expression, folic acid has been shown to indirectly stimulate PGC1 $\alpha$ activity to induce ROS clearance and mitochondrial biogenesis [163]. Parkin mutant files specifically showed improvements in lifespan and locomotor ability following folic acid supplementation. Interestingly, the neuroprotective effects of p53 repression have also been shown to be dependent on zinc levels. Zinc deficiency has been associated with p53 induced apoptosis and ROS induced DNA damage [164]. In folic acid treated parkin mutants, low p53 and high zinc levels were observed, suggesting that folic acid positively influences zinc metabolism. Furthermore, separate studies have shown that supplementation of zinc chloride has neuroprotective effects on the lifespan and locomotor ability of parkin mutant flies [165]. Alternative to transcription factor mediated defences, direct upregulation of antioxidant enzyme activity has been observed to improve phenotypes in pink1/parkin mutants following treatment with minocycline (antibiotic) and the phytoextract Mucuna pruriens $[166,167]$. The latter have been shown to scavenge free radicals by upregulating SOD and GSH activity.

Apart from the regulation of antioxidant enzymes, certain compounds have appeared to target dysfunctional ETS activity in the fight against ROS stress. Nicotine has been shown to promote complex I activity thus reducing electron leakage and ROS accumulation in parkin mutant flies [168]. From studies in pink1 mutant flies, Vitamin $\mathrm{K}_{2}$ has been shown to function as an electron carrier analogous to ubiquinone in order to promote oxidative phosphorylation [169]. With regard to mitochondrial biogenesis, certain compounds have been found to specifically upregulate chaperone mediated protein clearance and mitochondrial dynamics (mitophagy) in pink1 mutant flies [170, 171]. 
While these studies have provided promising results to explore their potential application in precision medicine, clinical trials of the dietary supplementation of compounds are needed to evaluate their true therapeutic value in patients.

\section{Discussion}

Fly models implement a wide variety of genetic tools (GAL4/UAS, RNAi) to perform unbiased genome-wide screening of PD-associated genes and mutations [17]. These methods help to understand the cellular consequences of mutations and gene overexpression/silencing in direct relation to disease phenotypes while also identifying additional factors that act in parallel/common cellular pathways to influence PD-pathology [15, 22]. Similar to post-mortem PD brains, Drosophila pink1/ parkin mutants replicate most pathological features such as abnormal mitochondrial morphology, protein aggregation and loss of dopaminergic neuron clusters $[19,80]$. The mechanisms behind such hallmarks have been extensively studied in flies, leading to some interesting results.

Starting with mitochondrial dynamics, the inhibition of mitochondrial fusion and upregulation of mitophagy has been observed to impede neural degeneration in both mammalian and fly models of PINK1/Parkin mutants. Specifically, the upregulation of the E3 ligase MUL1 halts the hyperfusion of damaged mitochondria while inhibition of Miro GTPase and de-ubiquitinases USP15/USP30 promotes their clearance via mitophagy $[62,74,76,79]$. In contrast, studies on mitochondrial fission have inferred diverging mechanisms for neuroprotection. Mammalian Parkin supposedly inhibits Drp-1 mediated fission to reduce the accumulation of injured mitochondria within neurons, while Drosophila pink1/ parkin mutants benefit from increased Drp-1fission via autophagy related gene Atg-1 and phosphoinositide Fwd kinase [65, 67, 68]. The latter mechanisms have suggested that neuron survival is prolonged in the event of increased ATP availability (sequestration of healthy mitochondria via Fwd-Drp-1 fission) and clearance of injured mitochondria (Drp-1 fission and subsequent mitophagy via Atg-1) (Fig. 1). Despite the previous observations made from mammalian studies of Parkin, the theory that upregulating Drp-1 mediated fission may decrease dopaminergic loss in humans has grown convincing. Particularly, a recent study involving $S N C A$ transgenic mice showed pronounced $\alpha$-synuclein pathology and mitochondrial enlargement as a consequence of decreased Drp-1 mediated fission [172]. Furthermore, these phenotypes were not observed in wild-type mice. Atg-1 and Fwd kinase currently remain as hypothetical targets for therapy; however it is tempting to speculate if their analysis in mammalian models will lead to similar results.
Multiple animal and in vitro studies have repeatedly shown impaired ROS metabolism to be at the epicentre of cellular dysfunction in PD [45]. By triggering unwanted protein aggregation, membrane instability, DNA damage and apoptotic cell death, the deleterious effects of ROS stress are endless. Free radical accumulation primarily stems from ETS dysfunction (particularly complex I) and antioxidant enzyme deficiency [97]. Looking into how ROS metabolism is affected by the PINK/Parkin axis, transgenic flies have shed light on multiple cellular pathways that can be induced to rescue ROS associated dopaminergic loss in pink1/parkin mutants (Fig. 1). Overexpression of UCP4, Trap1 and CHIP have been shown to rescue pink1/parkin phenotypes by respectively increasing free radical neutralization and complex I activity $[103,110,134]$. The exact mechanisms by which CHIP and Trap1 promote complex I activity are unclear; however, being members of the Hsp70/90 class of chaperone proteins it is likely that they might influence the reassembly of misfolded respiratory complexes and thereby impede electron leakage [132, 173]. Furthermore, studies in flies and mammalian cell lines have shown that CHIP is implicated in Parkin mediated proteasomal/aggresomal clearance of misfolded proteins that exert ER-stress, such as the GPCR Pael-R [133, 134]. The neuroprotective effects of CHIP in pink1/parkin mutants are thus twofold, by mitigating ROS stress and protein aggregation; two processes that are mutually exacerbating causes of dopaminergic death [174].

Alongside these pathways, upregulation of the transcription factors PGC1 $\alpha$ and FOXO, have been observed to increase the expression of antioxidant enzymes such as MnSOD, SOD2 and GPX1 $[105,112]$. Both fly and mammalian studies have demonstrated the neuroprotective activity of PGC1 $\alpha$ to ensue following PINK1/Parkin mediated degradation of the $\mathrm{KRAB} /$ zinc finger binding protein PARIS, its transcriptional repressor [113]. Thus, knockdown of PARIS ameliorates oxidative stress [115]. Furthermore, the induction of canonical Wnt2 signalling in pink1/parkin mutant flies upregulates PGC1 $\alpha$ and FOXO activity via $\beta$-catenin to spur antioxidant defences [120]. PGC1a and FOXO3 have also been associated with inhibiting $\alpha$-synuclein aggregation in vitro and in murine models, further cementing them as possible multi-purpose therapeutic targets $[175,176]$.

With regard to apoptotic cell death, studies in mammalian models have outlined clear roles for PINK1 and Parkin in regulating the intrinsic pathway. While PINK1 has been shown to induce apoptosis via phospho-activation of Bcl-xL under conditions of severe mitochondrial stress, Parkin ubiquitylates Bak to inhibit apoptosis in the event of acute mitochondrial damage [146, 147]. Interestingly, fly models have suggested that parkin switches 


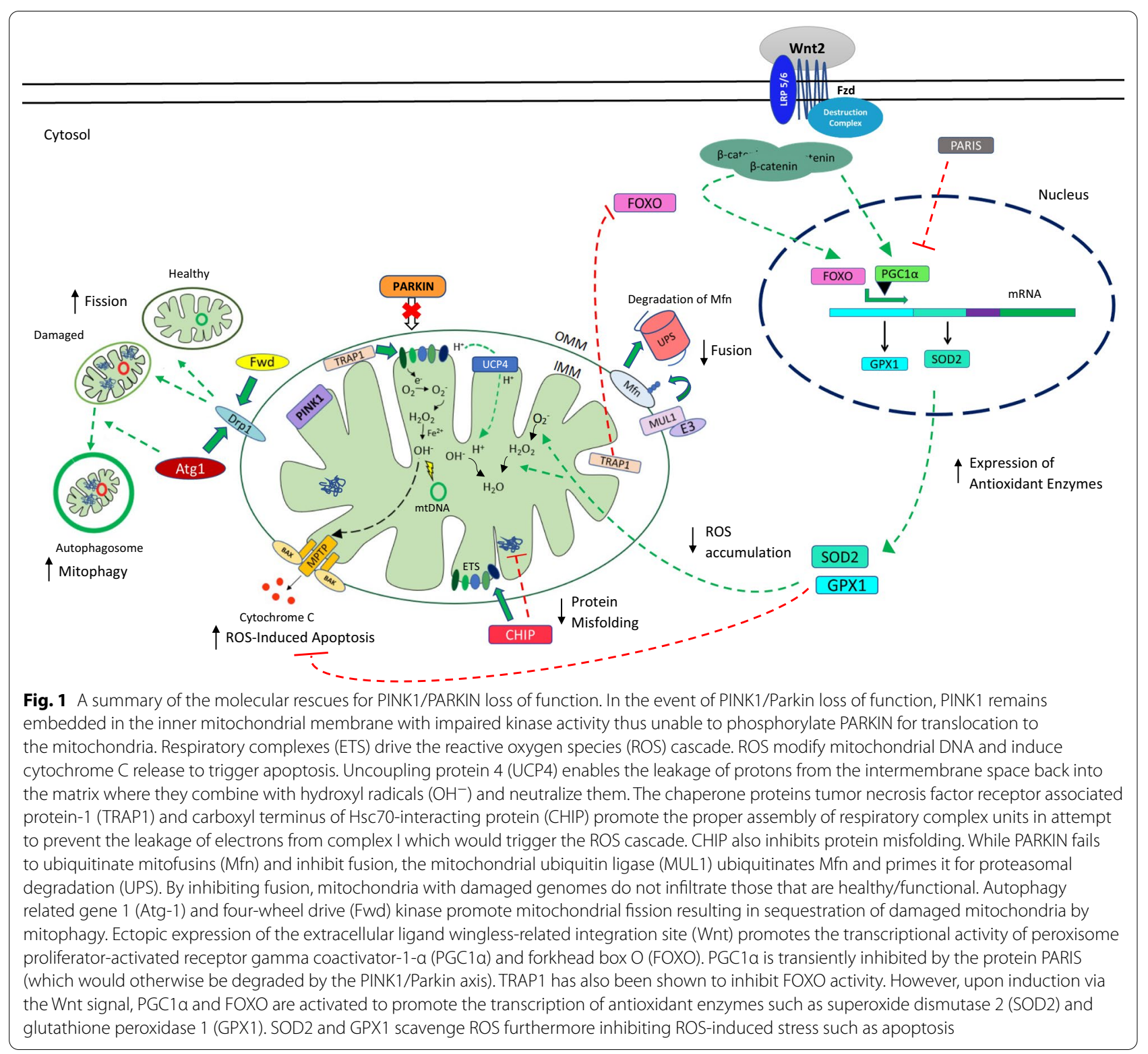

between mono- and polyubiquitination of VDAC1, an anion channel associated with the MPTP. Monoubiquitination of VDAC1 inhibits mitochondrial calcium overload and thereby apoptosis, whereas polyubiquitination of VDAC1 triggers mitophagy [154]. Although the cellular signals that influence the decision between mitophagy and apoptosis are unclear, these studies highlight that Parkin is an active sensor of fluctuating intracellular stress, adopting different mechanisms to maximize neural survival and evade premature death.

Considering the multiple factors that participate in the aggravation of dopaminergic death, a minor area of research has focused on studying the effect of specific compounds on mitochondrial dysfunction, oxidative stress, neuroinflammation and environmental toxin induced neurodegeneration [155, 177]. Along these lines, Drosophila PINK1/Parkin models have been employed to test the efficacy of various natural compounds against neural death and uncover how they target the aforesaid mechanisms (Table 1). Despite favourable results from fly studies, few clinical trials have sought to examine the effects of dietary supplementation of these compounds. The most recent clinical investigation on the latter is being based on testing the therapeutic effect of Vitamin $\mathrm{K}_{2}$ supplementation in patients with PINK1 mutations [178]. The Drosophila 
study preceding this investigation reported the survival of pink 1 mutants to have improved following upregulation of Heix (Drosophila ortholog of human UBIAD1), an enzyme that converts Vitamin $\mathrm{K}_{1}$ to $\mathrm{K}_{2}$. While the knockdown of Heix aggravated the phenotypes of pink1 mutants, they were rescued following the supplementation of Vitamin $K_{2}$ rich food [169]. Should the result of the ongoing clinical trial affirm the supposed benefit of Vitamin $K_{2}$, it would encourage further research on the clinical application of other compounds which fly models have drawn attention to.

Taken together, these studies have helped broaden our perspective of PD from a pathological and therapeutic standpoint.

\section{Conclusion}

The PINK1/Parkin axis governs a hoard of dynamic processes from mitochondrial biogenesis to protein clearance and ROS homeostasis all of which are critical in supporting the survival of neurons. Given the evolutionary conservation of genes between humans and flies, Drosophila melanogaster has served as a unique model to probe the complex cellular mechanisms that deter the health of dopaminergic neurons in PD. It is important to decipher such mechanisms not only for therapeutic purposes but to also understand why PINK1/Parkin loss of function phenotypes vary amongst different models of PD. Validation of these pathways in mammalian systems could refine the spectrum of focus for disease and nondisease modifying therapies while also reinforcing the importance of fly studies in PD.

\footnotetext{
Abbreviations

Atg-1: Autophagy related gene 1; Cdc37: Hsp90 co-chaperone Cdc37; CHIP: Carboxyl terminus of Hsc70-Interacting Protein; COMT: Catechol-O-Methyltransferase; DJ-1: Protein Deglycase; Drp1: Dynamin related protein 1; ETS: Electron Transport System; Fis 1: Fission-1 protein; FOXO: Forkhead box O; Fwd: Four-wheel drive; GAL4/UAS: Gal4 Transcription Factor/Upstream Activating Sequence; GPX1: Glutathione Peroxidase 1; GSH: Reduced glutathione; Hsp90: Heat shock protein 90; IMM: Inner Mitochondrial Membrane; LRRK2: LeucineRich Repeat Kinase 2; MAOB: Monoamine oxidase B; Mfn1: Mitofusin 1; Mfn2: Mitofusin 2; Miro: Mitochondrial Rho GTPase; MUL1: Mitochondrial Ubiquitin Ligase; Nrf2: Nuclear factor erythroid 2-related factor 2; OMM: Outer Mitochondrial Membrane; OMP: Outer Membrane Protein; OPA1: Optic Atrophy 1; PD: Parkinson's Disease; PGC1a: Peroxisome proliferator-activated receptor Gamma (PPARG) Coactivator-1-a; PINK1: PTEN-Induced Putative Kinase 1; RNAi: RNA interference; ROS: Reactive Oxygen Species; Sir2: Silent information regulator 2; SNCA: Alpha-Synuclein; SOD: Superoxide Dismutase; SUMO: Small Ubiquitin-Like Modifier;TRAK:Trafficking Kinesin Protein; Trap1:Tumor Necrosis Factor (TNF) receptor-associated protein 1; UCP4: Uncoupling Protein 4; UPR: Unfolded Protein Response; UPS: Ubiquitin-Proteasome System; USP15: Ubiquitin Specific Peptidase 15; USP30: Ubiquitin Specific Peptidase 30; USP8: Ubiquitin Specific Peptidase 8; VDAC1: Voltage-Dependent Anion Channel 1; VPS35: Vacuolar Protein Sorting Ortholog 35; Wnt: Wingless-related integration site.
}

Acknowledgements

Not applicable.

\section{Authors' contributions}

SG drafted the original manuscript and worked on the visualization. VDP contributed to conceptualization, writing, review editing and supervision. Both authors read and approved the final manuscript.

\section{Funding}

This research did not receive any specific grant from funding agencies in the public, commercial, or not-for-profit sectors.

Availability of data and materials

Not applicable.

\section{Declarations}

Ethics approval and consent to participate

Not applicable.

\section{Consent for publication}

Not applicable.

\section{Competing interests}

The authors have no competing interest to declare.

Received: 29 May 2021 Accepted: 15 October 2021

Published online: 26 November 2021

\section{References}

1. Moore DJ, West AB, Dawson VL, Dawson TM (2005) Molecular pathophysiology of Parkinson's disease. Annu Rev Neurosci 28:57-87. https:// doi.org/10.1146/annurev.neuro.28.061604.135718

2. Armstrong MJ, Okun MS (2020) Diagnosis and treatment of Parkinson disease: a review. JAMA J Am Med Assoc 323:548-560. https://doi.org/ 10.1001/jama.2019.22360

3. Marras C, Lang A (2013) Parkinson's disease subtypes: lost in translation? J Neurol Neurosurg Psychiatry 84:409-415. https://doi.org/10.1136/ jnnp-2012-303455

4. de Lau LM, Breteler MM (2006) Epidemiology of Parkinson's disease. Lancet Neurol 5:525-535. https://doi.org/10.1016/S1474-4422(06) 70471-9

5. Abbas MM, Xu Z, Tan LCS (2018) Epidemiology of Parkinson's diseaseeast versus west. Mov Disord Clin Pract 5:14-28. https://doi.org/10. $1002 /$ mdc3.12568

6. Schulte C, Gasser T (2011) Genetic basis of Parkinson's disease: inheritance, penetrance, and expression. Appl Clin Genet 4:67-80. https://doi. org/10.2147/TACG.S11639

7. MDSGene n.d. https://www.mdsgene.org/g4d. Accessed 24 May 2021

8. Surathi P, Jhunjhunwala K, Yadav R, Pal PK (2016) Research in Parkinson's disease in India: a review. Ann Indian Acad Neurol 19:9-20. https://doi. org/10.4103/0972-2327.167713

9. Muangpaisan W, Hori H, Brayne C (2009) Systematic review of the prevalence and incidence of Parkinson's disease in Asia. J Epidemiol. https://doi.org/10.2188/jea.JE20081034

10. Kasten M, Hartmann C, Hampf J, Schaake S, Westenberger A, Vollstedt EJ et al (2018) Genotype-phenotype relations for the Parkinson's disease genes Parkin, PINK1, DJ1: MDSGene systematic review. Mov Disord 33:730-741. https://doi.org/10.1002/mds.27352

11. Poewe W, Seppi K, Tanner CM, Halliday GM, Brundin P, Volkmann J et al (2017) Parkinson disease. Nat Rev Dis Primers 3:1-21. https://doi.org/10. 1038/nrdp.2017.13

12. Barodia SK, Creed RB, Goldberg MS (2017) Parkin and PINK1 functions in oxidative stress and neurodegeneration. Brain Res Bull 133:51-59. https://doi.org/10.1016/j.brainresbull.2016.12.004

13. Barazzuol L, Giamogante F, Brini M, Cali T (2020) PINK1/Parkin mediated mitophagy, Ca2+ signalling, and ER-Mitochondria contacts in Parkinson's Disease. Int J Mol Sci 21:1772. https://doi.org/10.3390/ijms210517 72 
14. Zhang CW, Hang L, Yao TP, Lim KL (2016) Parkin regulation and neurodegenerative disorders. Front Aging Neurosci 7:248. https://doi.org/10. 3389/fnagi.2015.00248

15. Paricio N, Muñoz-Soriano V (2011) Drosophila models of Parkinson's disease: discovering relevant pathways and novel therapeutic strategies. Parkinson's Dis. https://doi.org/10.4061/2011/520640

16. Potashkin JA, Blume SR, Runkle NK (2011) Limitations of animal models of Parkinson's disease. Parkinson's Dis. https://doi.org/10.4061/2011/ 658083

17. Dung VM, Thao DTP (2018) Parkinson's disease model. In: Yamaguchi M (ed) Advances in experimental medicine and biology, vol 1076. Springer, New York, pp 41-61. https://doi.org/10.1007/ 978-981-13-0529-0 4

18. Houlihan K (2020) CRISPR Caspase 9 strategies to explore parkin loss-offunction Drosophila development. FASEB J 34:1-1. https://doi.org/10. 1096/fasebj.2020.34.s1.09897

19. Bastioli G, Regoni M, Cazzaniga F, de Luca CMG, Bistaffa E, Zanetti L et al (2021) Animal models of autosomal recessive parkinsonism. Biomedicines 9:812. https://doi.org/10.3390/biomedicines9070812

20. Sang TK, Chang HY, Lawless GM, Ratnaparkhi A, Mee L, Ackerson LC et al (2007) A Drosophila model of mutant human parkin-induced toxicity demonstrates selective loss of dopaminergic neurons and dependence on cellular dopamine. J Neurosci 27:981-992. https://doi. org/10.1523/JNEUROSCI.4810-06.2007

21. Blandini F, Armentero M-T (2012) Animal models of Parkinson's disease. FEBS J 279:1156-1166. https://doi.org/10.1111/j.1742-4658.2012. 08491.x

22. Aryal B, Lee Y (2019) Disease model organism for Parkinson disease: Drosophila melanogaster. BMB Rep 52:250-258. https://doi.org/10. 5483/BMBRep.2019.52.4.204

23. Haruyama N, Cho A, Kulkarni AB (2009) Overview: engineering transgenic constructs and mice. Curr Protoc Cell Biol. https://doi.org/10. 1002/0471143030.cb1910s42

24. El-Brolosy MA, Stainier DYR (2017) Genetic compensation: a phenomenon in search of mechanisms. PLoS Genet 13:e1006780. https://doi. org/10.1371/journal.pgen.1006780

25. Jagmag SA, Tripathi N, Shukla SD, Maiti S, Khurana S (2016) Evaluation of models of Parkinson's disease. Front Neurosci 9:503. https://doi.org/ 10.3389/fnins.2015.00503

26. Dawson TM, Dawson VL (2010) The role of parkin in familial and sporadic Parkinson's disease. Mov Disord 25:S32-S39. https://doi.org/10. 1002/mds.22798

27. Kitada T, Asakawa S, Hattori N, Matsumine H, Yamamura Y, Minoshima S et al (1998) Mutations in the parkin gene cause autosomal recessive juvenile parkinsonism. Nature 392:605-608. https://doi.org/10.1038/ 33416

28. Klein C, Westenberger A (2012) Genetics of Parkinson's disease. Cold Spring Harbor Perspect Med 2:a008888. https://doi.org/10.1101/cshpe rspect.a008888

29. Panicker N, Dawson VL, Dawson TM (2017) Activation mechanisms of the E3 ubiquitin ligase parkin. Biochem J 474:3075-3086. https://doi. org/10.1042/BCJ20170476

30. Deshaies RJ, Joazeiro CAP (2009) RING domain E3 ubiquitin ligases. Annu Rev Biochem 78:399-434. https://doi.org/10.1146/annurev.bioch em.78.101807.093809

31. Tracz M, Bialek W (2021) Beyond K48 and K63: non-canonical protein ubiquitination. Cell Mol Biol Lett 26:1-17. https://doi.org/10.1186/ s11658-020-00245-6

32. Rogaeva E, Johnson J, Lang AE, Gulick C, Gwinn-Hardy K, Kawarai T et al (2004) Analysis of the PINK1 gene in a large cohort of cases with Parkinson Dis. Arch Neurol 61:1898-1904. https://doi.org/10.1001/archn eur.61.12.1898

33. Woodroof HI, Pogson JH, Begley M, Cantley LC, Deak M, Campbell DG et al (2011) Discovery of catalytically active orthologues of the Parkinson's disease kinase PINK1: analysis of substrate specificity and impact of mutations. Open Biol 1:110012. https://doi.org/10.1098/rsob.110012

34. Jin SM, Lazarou M, Wang C, Kane LA, Narendra DP, Youle RJ (2010) Mitochondrial membrane potential regulates PINK1 import and proteolytic destabilization by PARL. J Cell Biol 191:933-942. https://doi.org/10. 1083/jcb.201008084
35. Okatsu K, Oka T, Iguchi M, Imamura K, Kosako H, Tani N et al (2012) PINK1 autophosphorylation upon membrane potential dissipation is essential for Parkin recruitment to damaged mitochondria. Nat Commun. https://doi.org/10.1038/ncomms2016

36. Kane LA, Lazarou M, Fogel Al, Li Y, Yamano K, Sarraf SA et al (2014) PINK1 phosphorylates ubiquitin to activate parkin E3 ubiquitin ligase activity. J Cell Biol 205:143-153. https://doi.org/10.1083/jcb.201402104

37. Kim Y, Park J, Kim S, Song S, Kwon SK, Lee SH et al (2008) PINK1 controls mitochondrial localization of Parkin through direct phosphorylation. Biochem Biophys Res Commun 377:975-980. https://doi.org/10.1016/j. bbrc.2008.10.104

38. Weihofen A, Ostaszewski B, Minami Y, Selkoe DJ (2008) Pink1 Parkinson mutations, the $\mathrm{Cdc} 37 / \mathrm{Hsp} 90$ chaperones and Parkin all influence the maturation or subcellular distribution of Pink1. Hum Mol Genet 17:602-616. https://doi.org/10.1093/hmg/ddm334

39. Ando M, Fiesel FC, Hudec R, Caulfield TR, Ogaki K, Górka-Skoczylas P et al (2017) The PINK1 p.I368N mutation affects protein stability and ubiquitin kinase activity. Mol Neurodegener 12:1-19. https://doi.org/10. 1186/s13024-017-0174-Z

40. Clark IE, Dodson MW, Jiang C, Cao JH, Huh JR, Seol JH et al (2006) Drosophila pink1 is required for mitochondrial function and interacts genetically with parkin. Nature 441:1162-1166. https://doi.org/10.1038/ nature04779

41. Yang Y, Gehrke S, Imai Y, Huang Z, Ouyang Y, Wang JW et al (2006) Mitochondrial pathology and muscle and dopaminergic neuron degeneration caused by inactivation of Drosophila Pink1 is rescued by Parkin. Proc Natl Acad Sci USA 103:10793-10798. https://doi.org/10.1073/pnas. 0602493103

42. Park J, Lee SB, Lee S, Kim Y, Song S, Kim S et al (2006) Mitochondrial dysfunction in Drosophila PINK1 mutants is complemented by parkin. Nature 441:1157-1161. https://doi.org/10.1038/nature04788

43. Greene JC, Whitworth AJ, Kuo I, Andrews LA, Feany MB, Pallanck LJ (2003) Mitochondrial pathology and apoptotic muscle degeneration in Drosophila parkin mutants. Proc Natl Acad Sci USA 100:4078-4083. https://doi.org/10.1073/pnas.0737556100

44. Julienne H, Buhl E, Leslie DS, Hodge JJL (2017) Drosophila PINK1 and parkin loss-of-function mutants display a range of non-motor Parkinson's disease phenotypes. Neurobiol Dis 104:15-23. https://doi.org/10. 1016/j.nbd.2017.04.014

45. Eriksen JL, Wszolek Z, Petrucelli L (2005) Molecular pathogenesis of Parkinson Disease. Arch Neurol 62:353-357. https://doi.org/10.1001/ archneur.62.3.353

46. Langston JW, Ballard P, Tetrud JW, Irwin I (1983) Chronic Parkinsonism in humans due to a product of meperidine-analog synthesis. Science (New York, NY) 219:979-980. https://doi.org/10.1126/science.6823561

47. Ali SF, David SN, Newport GD, Cadet JL, Slikker W (1994) MPTP-induced oxidative stress and neurotoxicity are age-dependent: evidence from measures of reactive oxygen species and striatal dopamine levels. Synapse 18:27-34

48. Wang X, Su B, Liu W, He X, Gao Y, Castellani RJ et al (2011) DLP1dependent mitochondrial fragmentation mediates 1-methyl-4-phenylpyridinium toxicity in neurons: implications for Parkinson's disease. Aging Cell 10:807-823. https://doi.org/10.1111/j.1474-9726.2011. 00721.X

49. Su YC, Qi X (2013) Inhibition of excessive mitochondrialfissionreduced aberrant autophagy and neuronal damage caused by LRRK2 G2019S mutation. Hum Mol Genet 22:4545-4561. https://doi.org/10.1093/ hmg/ddt301

50. Haylett W, Swart C, van der Westhuizen F, van Dyk H, van der Merwe L, van der Merwe C et al (2016) Altered mitochondrial respiration and other features of mitochondrial function in parkin-mutant fibroblasts from Parkinson's disease patients. Parkinson's Dis 2016:1-11. https://doi. org/10.1155/2016/1819209

51. Hsieh CH, Shaltouki A, Gonzalez AE, Bettencourt da Cruz A, Burbulla LF, St. Lawrence E et al (2016) Functional impairment in miro degradation and mitophagy is a shared feature in familial and sporadic Parkinson's disease. Cell Stem Cell 19:709-724. https://doi.org/10.1016/j.stem.2016. 08.002

52. Trancikova A, Tsika E, Moore DJ (2012) Mitochondrial dysfunction in genetic animal models of Parkinson's disease. Antioxid Redox Signal 16:896-919. https://doi.org/10.1089/ars.2011.4200 
53. Mandal A, Drerup CM (2019) Axonal transport and mitochondrial function in neurons. Front Cell Neurosci 13:373. https://doi.org/10.3389/ fncel.2019.00373

54. DiMauro S, Schon EA (2003) Mitochondrial respiratory-chain diseases. N Engl J Med 348:2656-2668. https://doi.org/10.1056/NEJMra022567

55. Chen H, Chan DC (2009) Mitochondrial dynamics-fusion, fission, movement, and mitophagy —in neurodegenerative diseases. Hum Mol Genet 18:R169-R176. https://doi.org/10.1093/hmg/ddp326

56. Verstreken P, Ly CV, Venken KJT, Koh T-W, Zhou Y, Bellen HJ (2005) Synaptic mitochondria are critical for mobilization of reserve pool vesicles at Drosophila neuromuscular junctions. Neuron 47:365-378. https://doi. org/10.1016/j.neuron.2005.06.018

57. Brown MR, Sullivan PG, Geddes JW (2006) Synaptic Mitochondria are more susceptible to $\mathrm{Ca}^{2+}$ overload than nonsynaptic mitochondria *. J Biol Chem 281:11658-11668. https://doi.org/10.1074/jbc.M510303200

58. Detmer SA, Chan DC (2007) Functions and dysfunctions of mitochondrial dynamics. Nat Rev Mol Cell Biol 8:870-879. https://doi.org/10. 1038/nrm2275

59. Perier C, Vila M (2012) Mitochondrial biology and Parkinson's disease. Cold Spring Harbor Perspect Med 2:a009332. https://doi.org/10.1101/ cshperspect.a009332

60. Jin SM, Youle RJ (2013) The accumulation of misfolded proteins in the mitochondrial matrix is sensed by PINK1 to induce PARK2/Parkin-mediated mitophagy of polarized mitochondria. Autophagy 9:1750-1757. https://doi.org/10.4161/auto.26122

61. Xiao B, Goh JY, Xiao L, Xian H, Lim KL, Liou YC (2017) Reactive oxygen species trigger Parkin/PINK1 pathway — dependent mitophagy by inducing mitochondrial recruitment of Parkin. J Biol Chem 292:1669716708. https://doi.org/10.1074/jbc.M117.787739

62. Tanaka A, Cleland MM, Xu S, Narendra DP, Suen DF, Karbowski M et al (2010) Proteasome and p97 mediate mitophagy and degradation of mitofusins induced by Parkin. J Cell Biol 191:1367-1380. https://doi.org/ $10.1083 / \mathrm{jcb} .201007013$

63. Ziviani E, Tao RN, Whitworth AJ (2010) Drosophila Parkin requires PINK1 for mitochondrial translocation and ubiquitinates Mitofusin. Proc Natl Acad Sci USA 107:5018-5023. https://doi.org/10.1073/pnas.0913485107

64. Chang CR, Blackstone C (2010) Dynamic regulation of mitochondrial fission through modification of the dynamin-related protein Drp1. Ann NY Acad Sci 1201:34-39. https://doi.org/10.1111/j.1749-6632.2010. 05629.x

65. Wang H, Song P, Du L, Tian W, Yue W, Liu M et al (2011) Parkin ubiquitinates Drp1 for proteasome-dependent degradation: implication of dysregulated mitochondrial dynamics in Parkinson disease. J Biol Chem 286:11649-11658. https://doi.org/10.1074/jbc.M1 10.144238

66. Deng H, Dodson MW, Huang H, Guo M (2008) The Parkinson's disease genes pink1 and parkin promote mitochondrial fission and/or inhibit fusion in Drosophila. Proc Natl Acad Sci USA 105:14503-14508. https:// doi.org/10.1073/pnas.0803998105

67. Ma P, Yun J, Deng H, Guo M (2018) Atg1-mediated autophagy suppresses tissue degeneration in pink1/parkin mutants by promoting mitochondrial fission in Drosophila. Mol Biol Cell 29:3082-3092. https:// doi.org/10.1091/mbc.E18-04-0243

68. Terriente-Felix A, Wilson EL, Whitworth AJ (2020) Drosophila phosphatidylinositol-4 kinase fwd promotes mitochondrial fission and can suppress pink1/parkin phenotypes. PLoS Genet 16:e1008844. https:// doi.org/10.1371/journal.pgen.1008844

69. Yun J, Puri R, Yang H, Lizzio MA, Wu C, Sheng Z-H et al (2014) MUL1 acts in parallel to the PINK1/parkin pathway in regulating mitofusin and compensates for loss of PINK1/parkin. Elife. https://doi.org/10.7554/ elife.01958

70. Prudent J, Zunino R, Sugiura A, Mattie S, Shore GC, McBride HM (2015) MAPL SUMOylation of Drp1 stabilizes an ER/mitochondrial platform required for cell death. Mol Cell 59:941-955. https://doi.org/10.1016/j. molcel.2015.08.001

71. Taximaimaiti R, Li H (2019) MUL1 gene polymorphisms and Parkinson's disease risk. Acta Neurol Scand 139:483-487. https://doi.org/10.1111/ ane.13081

72. Heo JM, Ordureau A, Paulo JA, Rinehart J, Harper JW (2015) The PINK1PARKIN mitochondrial ubiquitylation pathway drives a program of OPTN/NDP52 recruitment and TBK1 activation to promote mitophagy. Mol Cell 60:7-20. https://doi.org/10.1016/j.molcel.2015.08.016
73. Durcan TM, Tang MY, Pérusse JR, Dashti EA, Aguileta MA, McLelland G et al (2014) USP 8 regulates mitophagy by removing K 6-linked ubiquitin conjugates from parkin. EMBO J 33:2473-2491. https://doi.org/10. 15252/embj.201489729

74. Cornelissen T, Haddad D, Wauters F, van Humbeeck C, Mandemakers W, Koentjoro B et al (2014) The deubiquitinase USP15 antagonizes Parkin-mediated mitochondrial ubiquitination and mitophagy. Hum Mol Genet 23:5227-5242. https://doi.org/10.1093/hmg/ddu244

75. Bingol B, Tea JS, Phu L, Reichelt M, Bakalarski CE, Song Q et al (2014) The mitochondrial deubiquitinase USP30 opposes parkin-mediated mitophagy. Nature 510:370-375. https://doi.org/10.1038/nature13418

76. Cunningham CN, Baughman JM, Phu L, Tea JS, Yu C, Coons M et al (2015) USP30 and parkin homeostatically regulate atypical ubiquitin chains on mitochondria. Nat Cell Biol 17:160-169. https://doi.org/10. 1038/ncb3097

77. Gersch M, Gladkova C, Schubert AF, Michel MA, Maslen S, Komander D (2017) Mechanism and regulation of the Lys6-selective deubiquitinase USP30. Nat Struct Mol Biol 24:920-930. https://doi.org/10.1038/nsmb. 3475

78. Sato Y, Okatsu K, Saeki Y, Yamano K, Matsuda N, Kaiho A et al (2017) Structural basis for specific cleavage of Lys6-linked polyubiquitin chains by USP30. Nat Struct Mol Biol 24:911-919. https://doi.org/10.1038/ nsmb.3469

79. Chakraborty J, Ziviani E (2020) Deubiquitinating enzymes in Parkinson's disease. Front Physiol 11:535. https://doi.org/10.3389/fphys.2020.00535

80. Braak H, Ghebremedhin E, Rüb U, Bratzke H, del Tredici K (2004) Stages in the development of Parkinson's disease-related pathology. Cell Tissue Res 318:121-134. https://doi.org/10.1007/s00441-004-0956-9

81. Hollenbeck PJ, Saxton WM (2005) The axonal transport of mitochondria. J Cell Sci 118:5411-5419. https://doi.org/10.1242/jcs.02745

82. Kay L, Pienaar IS, Cooray R, Black G, Soundararajan M (2018) Understanding Miro GTPases: implications in the treatment of neurodegenerative disorders. Mol Neurobiol 55:7352-7365. https://doi.org/10.1007/ s12035-018-0927-X

83. Tsai PI, Course MM, Lovas JR, Hsieh CH, Babic M, Zinsmaier KE et al (2014) PINK1-mediated phosphorylation of miro inhibits synaptic growth and protects dopaminergic neurons in drosophila. Sci Rep. https://doi.org/10.1038/srep06962

84. Wang X, Winter D, Ashrafi G, Schlehe J, Wong YL, Selkoe D et al (2011) PINK1 and Parkin target miro for phosphorylation and degradation to arrest mitochondrial motility. Cell 147:893-906. https://doi.org/10. 1016/j.cell.2011.10.018

85. Periñán MT, Gómez-Garre P, Blauwendraat C, Mir P, Bandres-Ciga S (2021) The role of RHOT1 and RHOT2 genetic variation on Parkinson disease risk and onset. Neurobiol Aging 97:144.e1-144.e3. https://doi. org/10.1016/j.neurobiolaging.2020.07.003

86. Liu S, Sawada T, Lee S, Yu W, Silverio G, Alapatt P et al (2012) Parkinson's disease-associated kinase PINK1 regulates miro protein level and axonal transport of mitochondria. PLoS Genet. https://doi.org/10.1371/journal. pgen. 1002537

87. Safiulina D, Kuum M, Choubey V, Gogichaishvili N, Liiv J, Hickey MA et al (2019) Miro proteins prime mitochondria for Parkin translocation and mitophagy. EMBO J. https://doi.org/10.15252/embj.201899384

88. Nguyen D, Bharat V, Conradson DM, Nandakishore P, Wang X (2021) Miro1 impairment in a Parkinson's at-risk cohort. Front Mol Neurosci. https://doi.org/10.3389/FNMOL.2021.734273

89. Vergara RC, Jaramillo-Riveri S, Luarte A, Moënne-Loccoz C, Fuentes $R$, Couve A et al (2019) The energy homeostasis principle: neuronal energy regulation drives local network dynamics generating behavior. Front Comput Neurosci 13:49. https://doi.org/10.3389/fncom.2019. 00049

90. Pathak D, Shields LY, Mendelsohn BA, Haddad D, Lin W, Gerencser AA et al (2015) The role of mitochondrially derived ATP in synaptic vesicle recycling. J Biol Chem 290:22325-22336. https://doi.org/10.1074/jbc. M115.656405

91. Chinta SJ, Andersen JK (2008) Redox imbalance in Parkinson's disease. Biochim Biophys Acta Gen Subj 1780:1362-1367. https://doi.org/10. 1016/j.bbagen.2008.02.005

92. Alam Zl, Jenner A, Daniel SE, Lees AJ, Cairns N, Marsden CD et al (1997) Oxidative DNA damage in the Parkinsonian brain: an apparent selective 
increase in 8-hydroxyguanine levels in substantia nigra. J Neurochem 69:1196-1203. https://doi.org/10.1046/j.1471-4159.1997.69031196.x

93. Yoritaka A, Hattori N, Uchida K, Tanaka M, Stadtman ER, Mizuno Y (1996) Immunohistochemical detection of 4-hydroxynonenal protein adducts in Parkinson disease. Proc Natl Acad Sci USA 93:2696-2701. https://doi. org/10.1073/pnas.93.7.2696

94. Keeney PM, Xie J, Capaldi RA, Bennett JP (2006) Parkinson's disease brain mitochondrial complex I has oxidatively damaged subunits and is functionally impaired and misassembled. J Neurosci 26:5256-5264. https://doi.org/10.1523/JNEUROSCI.0984-06.2006

95. Kösel S, Grasbon-Frodl EM, Mautsch U, Egensperger R, von Eitzen U, Frishman D et al (1998) Novel mutations of mitochondrial complex I in pathologically proven Parkinson disease. Neurogenetics 1:197-204. https://doi.org/10.1007/s100480050029

96. Hatcher JM, Pennell KD, Miller GW (2008) Parkinson's disease and pesticides: a toxicological perspective. Trends Pharmacol Sci 29:322-329. https://doi.org/10.1016/j.tips.2008.03.007

97. Orrenius S, Gogvadze V, Zhivotovsky B (2007) Mitochondrial oxidative stress: implications for cell death. Annu Rev Pharmacol Toxicol 47:143-183. https://doi.org/10.1146/annurev.pharmtox.47.120505. 105122

98. Circu ML, AwTY (2010) Reactive oxygen species, cellular redox systems, and apoptosis. Free Radical Biol Med 48:749-762. https://doi.org/10. 1016/j.freeradbiomed.2009.12.022

99. Valko M, Leibfritz D, Moncol J, Cronin MTD, Mazur M, Telser J (2007) Free radicals and antioxidants in normal physiological functions and human disease. Int J Biochem Cell Biol 39:44-84. https://doi.org/10.1016/j. biocel.2006.07.001

100. Morais VA, Verstreken P, Roethig A, Smet J, Snellinx A, Vanbrabant M et al (2009) Parkinson's disease mutations in PINK1 result in decreased Complex I activity and deficient synaptic function. EMBO Mol Med 1:99-111. https://doi.org/10.1002/emmm.200900006

101. Pridgeon JW, Olzmann JA, Chin LS, Li L (2007) PINK1 protects against oxidative stress by phosphorylating mitochondrial chaperone TRAP1. PLoS Biol 5:1494-1503. https://doi.org/10.1371/journal.pbio.0050172

102. Voos W, Röttgers K (2002) Molecular chaperones as essential mediators of mitochondrial biogenesis. Biochim Biophys Acta Mol Cell Res 1592:51-62. https://doi.org/10.1016/S0167-4889(02)00264-1

103. Costa AC, Loh SH, Martins LM (2013) Drosophila Trap1 protects against mitochondrial dysfunction in a PINK1/parkin model of Parkinson's disease. Cell Death Dis. https://doi.org/10.1038/cddis.2012.205

104. Kim H, Yang J, Kim MJ, Choi S, Chung JR, Kim JM et al (2016) Tumor necrosis factor receptor-associated protein 1 (TRAP1) mutation and TRAP1 inhibitor gamitrinibtriphenylphosphonium (G-TPP) induce a forkhead box O (FOXO)-dependent cell protective signal from mitochondria. J Biol Chem 291:1841-1853. https://doi.org/10.1074/jbc. M115.656934

105. Kim S, Koh H (2017) Role of FOXO transcription factors in crosstalk between mitochondria and the nucleus. J Bioenerg Biomembr 49:335-341. https://doi.org/10.1007/s10863-017-9705-0

106. Koh H, Kim H, Kim MJ, Park J, Lee HJ, Chung J (2012) Silent information regulator 2 (Sir2) and forkhead box O (FOXO) complement mitochondrial dysfunction and dopaminergic neuron loss in Drosophila PTENinduced kinase 1 (PINK1) null mutant. J Biol Chem 287:12750-12758. https://doi.org/10.1074/jbc.M111.337907

107. Ramsden DB, Ho PWL, Ho JWM, Liu HF, So DHF, Tse HM et al (2012) Human neuronal uncoupling proteins 4 and 5 (UCP4 and UCP5): structural properties, regulation, and physiological role in protection against oxidative stress and mitochondrial dysfunction. Brain Behav 2:468-478. https://doi.org/10.1002/brb3.55

108. Echtay KS, Pakay JL, Esteves TC, Brand MD (2005) Hydroxynonenal and uncoupling proteins: a model for protection against oxidative damage. BioFactors 24:119-130. https://doi.org/10.1002/biof.5520240114

109. Pfeiffer M, Kayzer EB, Yang X, Abramson E, Kenaston MA, Lago CU et al (2011) Caenorhabditis elegans UCP4 protein controls complex IImediated oxidative phosphorylation through succinate transport. J Biol Chem 286:37712-37720. https://doi.org/10.1074/jbc.M111.271452

110. Wu K, Liu J, Zhuang N, Wang T (2014) UCP4A protects against mitochondrial dysfunction and degeneration in pink1/parkin models of Parkinson's disease. FASEB J 28:5111-5121. https://doi.org/10.1096/f. 14-255802
111. Xu S, Yang X, Qian Y, Xiao Q (2018) Parkinson's disease-related DJ-1 modulates the expression of uncoupling protein 4 against oxidative stress. J Neurochem 145:312-322. https://doi.org/10.1111/jnc.14297

112. Austin S, St-Pierre J (2012) PGC1 a and mitochondrial metabolismemerging concepts and relevance in ageing and neurodegenerative disorders. J Cell Sci 125:4963-4971. https://doi.org/10.1242/jcs.113662

113. Shin JH, Ko HS, Kang H, Lee Y, Lee Y-I, Pletinkova O et al (2011) PARIS (ZNF746) repression of PGC-1 a contributes to neurodegeneration in Parkinson's disease. Cell 144:689-702. https://doi.org/10.1016/j.cell. 2011.02.010

114. Lee Y, Stevens DA, Kang SU, Jiang H, Lee Y-I, Ko HS et al (2017) PINK1 primes Parkin-mediated ubiquitination of PARIS in dopaminergic neuronal survival. Cell Rep 18:918-932. https://doi.org/10.1016/j.celrep. 2016.12.090

115. Pirooznia SK, Yuan C, Khan MR, Karuppagounder SS, Wang L, Xiong Y et al (2020) PARIS induced defects in mitochondrial biogenesis drive dopamine neuron loss under conditions of parkin or PINK1 deficiency. Mol Neurodegener. https://doi.org/10.1186/s13024-020-00363-x

116. Wareski P, Vaarmann A, Choubey V, Safiulina D, Liiv J, Kuum M et al (2009) PGC-1 a and PGC-1 $\beta$ regulate mitochondrial density in neurons. J Biol Chem 284:21379-21385. https://doi.org/10.1074/jbc.M109.018911

117. Sousa KM, Carlos Villaescusa J, Cajanek L, Ondr JK, Castelo-Branco G, Hofstra W et al (2010) Wnt2 regulates progenitor proliferation in the developing ventral midbrain. J Biol Chem 285:7246-7253. https://doi. org/10.1074/jbc.M109.079822

118. He CW, Liao CP, Pan CL (2018) Wnt signalling in the development of axon, dendrites and synapses. Open Biol. https://doi.org/10.1098/rsob. 180116

119. Berwick DC, Harvey K (2012) The importance of Wnt signalling for neurodegeneration in Parkinson's disease. Biochem Soc Trans 40:11231128. https://doi.org/10.1042/BST20120122

120. Xia SR, Wen XY, Fan XL, Chen XR, Wei ZW, Li QH et al (2020) Wnt2 overexpression protects against PINK1 mutant-induced mitochondrial dysfunction and oxidative stress. Mol Med Rep 21:2633-2641. https:// doi.org/10.3892/mmr.2020.11066

121. Essers MAG, de Vries-Smits LMM, Barker N, Polderman PE, Burgering BMT, Korswagen HC (2005) Functional interaction between $\beta$-catenin and FOXO in oxidative stress signaling. Science 308:1181-1184. https:// doi.org/10.1126/science.1109083

122. Rawal N, Corti O, Sacchetti P, Ardilla-Osorio H, Sehat B, Brice A et al (2009) Parkin protects dopaminergic neurons from excessive Wnt/ $\beta$ catenin signaling. Biochem Biophys Res Commun 388:473-478. https:// doi.org/10.1016/j.bbrc.2009.07.014

123. Dev KK, van der Putten H, Sommer B, Rovelli G (2003) Part I: Parkin-associated proteins and Parkinson's disease. Neuropharmacology 45:1-13. https://doi.org/10.1016/S0028-3908(02)00337-4

124. Vilchez D, Saez I, Dillin A (2014) The role of protein clearance mechanisms in organismal ageing and age-related diseases. Nat Commun 5:1-13. https://doi.org/10.1038/ncomms6659

125. Sweeney P, Park H, Baumann M, Dunlop J, Frydman J, Kopito R et al (2017) Protein misfolding in neurodegenerative diseases: implications and strategies. Transl Neurodegener 6:1-13. https://doi.org/10.1186/ s40035-017-0077-5

126. Waxman EA, Giasson BI (2009) Molecular mechanisms of a-synuclein neurodegeneration. Biochim Biophys Acta Mol Basis Dis 1792:616-624. https://doi.org/10.1016/j.bbadis.2008.09.013

127. Olzmann JA, Chin LS (2008) Parkin-mediated K63-linked polyubiquitination: a signal for targeting misfolded proteins to the aggresomeautophagy pathway. Autophagy 4:85-87. https://doi.org/10.4161/auto. 5172

128. Olzmann JA, Li A, Chudaev MV, Chen J, Perez FA, Palmiter RD et al (2007) Parkin-mediated K63-linked polyubiquitination targets misfolded DJ-1 to aggresomes via binding to HDAC6. J Cell Biol 178:1025-1038. https://doi.org/10.1083/jcb.200611128

129. Lim KL, Dawson VL, Dawson TM (2006) Parkin-mediated lysine 63-linked polyubiquitination: a link to protein inclusions formation in Parkinson's and other conformational diseases? Neurobiol Aging 27:524-529. https://doi.org/10.1016/j.neurobiolaging.2005.07.023

130. Sano R, Reed JC (2013) ER stress-induced cell death mechanisms. Biochim Biophys Acta Mol Cell Res 1833:3460-3470. https://doi.org/10. 1016/j.bbamcr.2013.06.028 
131. Imai Y, Soda M, Inoue H, Hattori N, Mizuno Y, Takahashi R (2001) An unfolded putative transmembrane polypeptide, which can lead to endoplasmic reticulum stress, is a substrate of Parkin. Cell 105:891-902. https://doi.org/10.1016/S0092-8674(01)00407-X

132. Murata S, Minami Y, Minami M, Chiba T, Tanaka K (2001) CHIP is a chaperone-dependent E3 ligase that ubiquitylates unfolded protein. EMBO Rep 2:1133-1138. https://doi.org/10.1093/embo-reports/kve246

133. Imai Y, Soda M, Hatakeyama S, Akagi T, Hashikawa T, Nakayama Kl et al (2002) CHIP is associated with Parkin, a gene responsible for familial Parkinson's Disease, and enhances its ubiquitin ligase activity. Mol Cell 10:55-67. https://doi.org/10.1016/S1097-2765(02)00583-X

134. Chen J, Xue J, Ruan J, Zhao J, Tang B, Duan R (2017) Drosophila CHIP protects against mitochondrial dysfunction by acting downstream of Pink1 in parallel with Parkin. FASEB J 31:5234-5245. https://doi.org/10. 1096/f.201700011R

135. Michel PP, Hirsch EC, Hunot S (2016) Understanding dopaminergic cell death pathways in Parkinson disease. Neuron 90:675-691. https://doi. org/10.1016/j.neuron.2016.03.038

136. Tompkins MM, Basgall EJ, Zamrini E, Hill WD (1997) Apoptotic-like changes in Lewy-body-associated disorders and normal aging in substantia nigral neurons. Am J Pathol 150:119

137. Anglade P, Vyas S, Javoy-Agid F, Herrero MT, Michel PP, Marquez J et al (1997) Histology and histopathology from cell biology to tissue engineering apoptosis and autophagy in nigral neurons of patients with Parkinson's disease. Histol Histopathol 12:25-31. https://doi.org/ 10.14670/HH-12.25

138. Viswanath V, Wu Y, Boonplueang R, Chen S, Stevenson FF, Yantiri F et al (2001) Caspase-9 activation results in downstream Caspase-8 activation and bid cleavage in 1-methyl-4-phenyl-1,2,3,6-tetrahydropyridineinduced Parkinson's disease. J Neurosci 21:9519. https://doi.org/10. 1523/JNEUROSCI.21-24-09519.2001

139. Mogi M, Togari A, Kondo T, Mizuno Y, Komure O, Kuno S et al (2000) Caspase activities and tumor necrosis factor receptor R1 (p55) level are elevated in the substantia nigra from Parkinsonian brain. J Neural Transm 107:335-341. https://doi.org/10.1007/s007020050028

140. Lev N, Melamed E, Offen D (2003) Apoptosis and Parkinson's disease. Prog Neuropsychopharmacol Biol Psychiatry 27:245-250. https://doi. org/10.1016/S0278-5846(03)00019-8

141. Macchi B, Paola R, Marino-Merlo F, Felice M, Cuzzocrea S, Mastino A (2015) Inflammatory and cell death pathways in brain and peripheral blood in Parkinson's disease. CNS Neurol Disord Drug Targets 14:313-324. https://doi.org/10.2174/1871527314666150225124928

142. Singh S, Kumar S, Dikshit M (2010) Involvement of the mitochondrial apoptotic pathway and nitric oxide synthase in dopaminergic neuronal death induced by 6-hydroxydopamine and lipopolysaccharide. Redox Rep 15:115-122. https://doi.org/10.1179/174329210X12650506623447

143. Li N, Ragheb K, Lawler G, Sturgis J, Rajwa B, Melendez JA et al (2003) Mitochondrial complex I inhibitor rotenone induces apoptosis through enhancing mitochondrial reactive oxygen species production *. J Biol Chem 278:8516-8525. https://doi.org/10.1074/jbc.M210432200

144. Eberhardt O, Schulz JB (2003) Apoptotic mechanisms and antiapoptotic therapy in the MPTP model of Parkinson's disease. Toxicol Lett 139:135-151. https://doi.org/10.1016/S0378-4274(02)00428-9

145. Ben-Shachar D, Zuk R, Glinka Y (1995) Dopamine neurotoxicity: inhibition of mitochondrial respiration. J Neurochem 64:718-723. https://doi. org/10.1046/j.1471-4159.1995.64020718.x

146. Bernardini JP, Brouwer JM, Tan IK, Sandow JJ, Huang S, Stafford CA et al (2019) Parkin inhibits BAK and BAX apoptotic function by distinct mechanisms during mitophagy. EMBO J. https://doi.org/10.1525/embj. 201899916

147. Arena G, Gelmetti V, Torosantucci L, Vignone D, Lamorte G, de Rosa P et al (2013) PINK1 protects against cell death induced by mitochondrial depolarization, by phosphorylating $\mathrm{BCl}-\mathrm{xL}$ and impairing its pro-apoptotic cleavage. Cell Death Differ 20:920-930. https://doi.org/10.1038/ cdd.2013.19

148. Westphal D, Dewson G, Czabotar PE, Kluck RM (2011) Molecular biology of Bax and Bak activation and action. Biochim Biophys Acta Mol Cell Res 1813:521-531. https://doi.org/10.1016/j.bbamcr.2010.12.019

149. Annis MG, Soucie EL, Dlugosz PJ, Cruz-Aguado JA, Penn LZ, Leber B et al (2005) Bax forms multispanning monomers that oligomerize to permeabilize membranes during apoptosis. EMBO J 24:2096-2103. https://doi.org/10.1038/sj.emboj.7600675

150. Bratton SB, Salvesen GS (2010) Regulation of the Apaf-1-caspase-9 apoptosome. J Cell Sci 123:3209-3214. https://doi.org/10.1242/jcs. 073643

151. Korsmeyer SJ, Wei MC, Saito M, Weiler S, Oh KJ, Schlesinger PH (2000) Pro-apoptotic cascade activates BID, which oligomerizes BAK or BAX into pores that result in the release of cytochrome c. Cell Death Differ 7:1166-1173. https://doi.org/10.1038/sj.cdd.4400783

152. Ren D, Tu H-C, Kim H, Wang GX, Bean GR, Takeuchi O et al (2010) BID, $B I M$, and PUMA are essential for activation of the BAX- and BAKdependent cell death program. Science (New York, NY) 330:1390-1393. https://doi.org/10.1126/science.1190217

153. Tsujimoto Y, Shimizu S (2000) VDAC regulation by the $\mathrm{BCl}-2$ family of proteins. Cell Death Differ 7:1174-1181. https://doi.org/10.1038/sj.cdd. 4400780

154. Ham SJ, Lee D, Yoo H, Jun K, Shin H, Chung J (2020) Decision between mitophagy and apoptosis by Parkin via VDAC1 ubiquitination. Proc Natl Acad Sci USA 117:4281-4291. https://doi.org/10.1073/pnas.1909814117

155. Whitworth AJ, Wes PD, Pallanck LJ (2006) Drosophila models pioneer a new approach to drug discovery for Parkinson's disease. Drug Discov Today 11:119-126. https://doi.org/10.1016/S1359-6446(05)03693-7

156. Auluck PK, Bonini NM (2002) Pharmacological prevention of Parkinson disease in Drosophila. Nat Med 8:1185-1186. https://doi.org/10.1038/ nm1102-1185

157. Auluck PK, Meulener MC, Bonini NM (2005) Mechanisms of suppression of a-synuclein neurotoxicity by geldanamycin in Drosophila. J Biol Chem 280:2873-2878. https://doi.org/10.1074/jbc.M412106200

158. Giuliano C, Cerri S, Blandini F (2021) Potential therapeutic effects of polyphenols in Parkinson's disease: in vivo and in vitro pre-clinical studies. Neural Regen Res 16:234-241. https://doi.org/10.4103/1673-5374. 290879

159. Singh CK, Chhabra G, Ndiaye MA, Garcia-Peterson LM, MacK NJ, Ahmad $\mathrm{N}$ (2018) The role of sirtuins in antioxidant and redox signaling. Antioxid Redox Signal 28:643-661. https://doi.org/10.1089/ars.2017.7290

160. Bonilla-Ramirez L, Jimenez-Del-Rio M, Velez-Pardo C (2013) Low doses of paraquat and polyphenols prolong life span and locomotor activity in knock-down parkin Drosophila melanogaster exposed to oxidative stress stimuli: implication in autosomal recessive juvenile Parkinsonism. Gene 512:355-363. https://doi.org/10.1016/j.gene.2012.09.120

161. Trinh K, Moore K, Wes PD, Muchowski PJ, Dey J, Andrews L et al (2008) Induction of the phase II detoxification pathway suppresses neuron loss in Drosophila models of Parkinson's disease. J Neurosci 28:465-472. https://doi.org/10.1523/JNEUROSCI.4778-07.2008

162. Boddupalli S, Mein JR, Lakkanna S, James DR (2012) Induction of phase 2 antioxidant enzymes by broccoli sulforaphane: perspectives in maintaining the antioxidant activity of vitamins A, C, and E. Front Genet. https://doi.org/10.3389/fgene.2012.00007

163. Srivastav S, Singh SK, Yadav AK, Srikrishna S (2015) Folic acid supplementation rescues anomalies associated with knockdown of parkin in dopaminergic and serotonergic neurons in Drosophila model of Parkinson's disease. Biochem Biophys Res Commun 460:780-785. https://doi. org/10.1016/j.bbrc.2015.03.106

164. Morita A, Ariyasu S, Ohya S, Takahashi I, Wang B, Tanaka K et al (2013) Evaluation of Zinc(II) chelators for inhibiting p53-mediated apoptosis. Oncotarget 4:2439-2450

165. Saini N, Schaffner W (2010) Zinc supplement greatly improves the condition of parkin mutant Drosophila. Biol Chem 391:513-518. https:// doi.org/10.1515/BC.2010.052

166. Ortega-Arellano HF, Jimenez-Del-Rio M, Velez-Pardo C (2017) Minocycline protects, rescues and prevents knockdown transgenic parkin Drosophila against paraquat/iron toxicity: implications for autosomic recessive juvenile parkinsonism. Neurotoxicology 60:42-53. https://doi. org/10.1016/j.neuro.2017.03.002

167. Baroli B, Loi E, Solari P, Kasture A, Moi L, Muroni P et al (2019) Evaluation of oxidative stress mechanisms and the effects of phytotherapic extracts on Parkinson's disease Drosophila PINK1B9 model. FASEB J 33:11028-11034. https://doi.org/10.1096/f.201901010

168. Chambers RP, Call GB, Meyer D, Smith J, Techau JA, Pearman K et al (2013) Nicotine increases lifespan and rescues olfactory and motor 
deficits in a Drosophila model of Parkinson's disease. Behav Brain Res 253:95-102. https://doi.org/10.1016/j.bbr.2013.07.020

169. Vos M, Esposito G, Edirisinghe JN, Vilain S, Haddad DM, Slabbaert JR et al (2012) Vitamin K2 is a mitochondrial electron carrier that rescues pink1 deficiency. Science 336:1306-1310. https://doi.org/10.1126/scien ce.1218632

170. Wu Z, Wu A, Dong J, Sigears A, Lu B (2018) Skin extract improves muscle function and extends lifespan of a Drosophila model of Parkinson's disease through activation of mitophagy. Exp Gerontol 113:10-17. https:// doi.org/10.1016/j.exger.2018.09.014

171. Liu M, Yu S, Wang J, Qiao J, Liu Y, Wang S et al (2020) Ginseng protein protects against mitochondrial dysfunction and neurodegeneration by inducing mitochondrial unfolded protein response in Drosophila melanogaster PINK1 model of Parkinson's disease. J Ethnopharmacol. https://doi.org/10.1016/j.jep.2019.112213

172. Portz P, Lee MK (2021) Changes in Drp1 function and mitochondrial morphology are associated with the a-Synuclein pathology in a transgenic mouse model of Parkinson's disease. Cells. https://doi.org/10. 3390/cells 10040885

173. Altieri DC, Stein GS, Lian JB, Languino LR (2012) TRAP-1, the mitochondrial Hsp90. Biochem Biophys Acta 1823:767-773. https://doi.org/10. 1016/j.bbamcr.2011.08.007

174. Lévy E, el Banna N, Baïlle D, Heneman-Masurel A, Truchet S, Rezaei H et al (2019) Causative links between protein aggregation and oxidative stress: a review. Int J Mol Sci. https://doi.org/10.3390/ijms20163896

175. Eschbach J, von Einem B, Müller K, Bayer H, Scheffold A, Morrison BE et al (2015) Mutual exacerbation of peroxisome proliferator-activated receptor $\psi$ coactivator 1a deregulation and a-synuclein oligomerization. Ann Neurol 77:15-32. https://doi.org/10.1002/ana.24294

176. Pino E, Amamoto R, Zheng L, Cacquevel M, Sarria J-C, Knott GW et al (2013) FOXO3 determines the accumulation a-synuclein and controls the fate of dopaminergic neurons in the substantia nigr. Hum Mol Genet 23:1435-1452

177. Maitra U, Ciesla L (2019) Using: Drosophila as a platform for drug discovery from natural products in Parkinson's disease. MedChemComm 10:867-879. https://doi.org/10.1039/c9md00099b

178. Prasuhn J, Kasten M, Vos M, König IR, Schmid SM, Wilms B et al (2021) The use of vitamin K2 in patients with Parkinson's disease and Mitochondrial Dysfunction (PD-K2): a theranostic pilot study in a placebocontrolled parallel group design. Front Neurol 11:592104. https://doi. org/10.3389/fneur.2020.592104

179. Cormier A, Morin C, Zini R, Tillement JP, Lagrue G (2001) In vitro effects of nicotine on mitochondrial respiration and superoxide anion generation. Brain Res 900:72-79. https://doi.org/10.1016/50006-8993(01) 02254-5

180. Wang D, Qian L, Xiong H, Liu J, Neckameyer WS, Oldham S et al (2006) Antioxidants protect PINK1-dependent dopaminergic neurons in Drosophila. Proc Natl Acad Sci USA 103:13520-13525. https://doi.org/ 10.1073/pnas.0604661103

\section{Publisher's Note}

Springer Nature remains neutral with regard to jurisdictional claims in published maps and institutional affiliations.

\section{Submit your manuscript to a SpringerOpen ${ }^{\odot}$ journal and benefit from:}

- Convenient online submission

- Rigorous peer review

- Open access: articles freely available online

- High visibility within the field

- Retaining the copyright to your article

Submit your next manuscript at $\boldsymbol{\nabla}$ springeropen.com 